\title{
COMPARATIVE ASYMPTOTICS FOR PERTURBED ORTHOGONAL POLYNOMIALS
}

\author{
FRANZ PEHERSTORFER AND ROBERT STEINBAUER
}

\begin{abstract}
Let $\left\{\Phi_{n}\right\}_{n \in \mathbb{N}_{0}}$ and $\left\{\widetilde{\Phi}_{n}\right\}_{n \in \mathbb{N}_{0}}$ be such systems of orthonormal polynomials on the unit circle that the recurrence coefficients of the perturbed polynomials $\widetilde{\Phi}_{n}$ behave asymptotically like those of $\Phi_{n}$. We give, under weak assumptions on the system $\left\{\Phi_{n}\right\}_{n \in \mathbb{N}_{0}}$ and the perturbations, comparative asymptotics as for $\widetilde{\Phi}_{n}^{*}(z) / \Phi_{n}^{*}(z)$ etc., $\Phi_{n}^{*}(z):=z^{n} \bar{\Phi}_{n}\left(\frac{1}{z}\right)$, on the open unit disk and on the circumference mainly off the support of the measure $\sigma$ with respect to which the $\Phi_{n}$ 's are orthonormal. In particular these results apply if the comparative system $\left\{\Phi_{n}\right\}_{n \in \mathbb{N}_{0}}$ has a support which consists of several arcs of the unit circumference, as in the case when the recurrence coefficients are (asymptotically) periodic.
\end{abstract}

\section{INTRODUCTION AND NOTATION}

Throughout this paper let $\left\{P_{n}\right\}_{n \in \mathbb{N}_{0}}$ be a sequence in $\mathbb{P}_{n}^{\mathbb{C}}$ (where $\mathbb{P}_{n}^{\mathbb{C}}$ denotes the set of complex polynomials with degree less or equal to $n$ ) generated by a recurrence relation of the form

$$
P_{n+1}(z)=z P_{n}(z)-\bar{a}_{n} P_{n}^{*}(z), \quad n \in \mathbb{N}_{0}, P_{0}(z)=1 .
$$

where

$$
a_{n} \in \mathbb{C} \text { and }\left|a_{n}\right|<1 .
$$

The parameters $a_{n}$ are called recurrence coefficients or Schur-parameters. In (1.1) $P_{n}^{*}$ denotes the reciprocal polynomial of $P_{n}$ defined by

$$
P_{n}^{*}(z)=z^{n} \bar{P}_{n}\left(\frac{1}{z}\right) .
$$

It is well known $[6, \S 11,13]$ that because of $(1.2)$ there exists a distribution function $\sigma$ (i.e. $\sigma$ is bounded, nondecreasing with an infinite set of points of increase) with respect to which the $P_{n}$ 's are orthogonal, i.e.

$$
\int_{0}^{2 \pi} e^{-i j \varphi} P_{n}\left(e^{i \varphi}\right) d \sigma(\varphi)=0 \text { for } j=0, \ldots, n-1 .
$$

Received by the editors March 5, 1994 and, in revised form, January 5, 1995 1991 Mathematics Subject Classification. Primary 42C05.

Key words and phrases. Orthogonal polynomials, unit circle, arcs, asymptotics.

Supported by the Austrian Fonds zur Förderung der wissenschaftlichen Forschung, Projektnummer P9267-PHY. 
In addition to (1.2) we assume henceforth that

$$
\varlimsup_{n \rightarrow \infty}\left|a_{n}\right|<1,
$$

which is by [20, Lemma 4, p. 110] (compare also [6, Theorem 19.1 (2)]) always fulfilled, if $\sigma$ is nonsingular.

If we set

$$
\Phi_{n}(z):=\frac{P_{n}(z)}{\sqrt{c_{0} d_{n}}}
$$

where $c_{0}:=\frac{1}{2 \pi} \int_{0}^{2 \pi} d \sigma(\varphi) \in \mathbb{R}^{+}$(note [6, Theorem 13.3] and (1.2)) and

$$
d_{n}^{(m)}:=\left\{\begin{array}{l}
\prod_{j=0}^{n-1}\left(1-\left|a_{j+m}\right|^{2}\right), \quad n \in \mathbb{N}, \quad m \in \mathbb{N}_{0}, \\
1, \quad n=0,
\end{array}\right.
$$

(we write $d_{n}$ instead of $d_{n}^{(0)}$ ), then the $\Phi_{n}$ 's are orthonormal by $[6,(2.7)$ and (4.2)], i.e.

$$
\frac{1}{2 \pi} \int_{0}^{2 \pi} \Phi_{n}\left(e^{i \varphi}\right) \overline{\Phi_{m}\left(e^{i \varphi}\right)} d \sigma(\varphi)=\delta_{n m}
$$

To the distribution function $\sigma$ we associate the function

$$
F(z):=F(z, \sigma):=\frac{1}{2 \pi c_{0}} \int_{0}^{2 \pi} \frac{e^{i \varphi}+z}{e^{i \varphi}-z} d \sigma(\varphi),
$$

which is analytic and pseudopositive (i.e. $\operatorname{Re} F(z)>0$ ) on $|z|<1$ and satisfies $F(0)=1$ (cf. $[6, \S 11]$ ). $F$ is called a Carathéodory-function (abbreviated C-function) with respect to $\sigma$.

Further $\left\{\widetilde{P}_{n}\right\}_{n \in \mathbb{N}_{0}}$ denotes henceforth another sequence of orthogonal polynomials on the unit circle satisfying the recurrence relation

$$
\widetilde{P}_{n+1}(z)=z \widetilde{P}_{n}(z)-\bar{b}_{n} \widetilde{P}_{n}^{*}(z), \quad n \in \mathbb{N}_{0}, \widetilde{P}_{0}(z)=1,
$$

where we assume, as in (1.2), that

$$
\left|b_{n}\right|<1, \quad n \in \mathbb{N}_{0} .
$$

Let $\tilde{\sigma}$ be the associated distribution function and $\widetilde{\Phi}_{n}^{\mathrm{ON}}, n \in \mathbb{N}_{0}$, the orthonormal polynomials. As in (1.5) we denote (for technical reasons we choose the norming factor $1 / \sqrt{c_{0} d_{n}}, d_{n}$ from (1.6))

$$
\widetilde{\Phi}_{n}(z):=\frac{\widetilde{P}_{n}(z)}{\sqrt{c_{0} d_{n}}} .
$$

Note that in general $\widetilde{\Phi}_{n}$ is not an orthonormal polynomial, i.e. $\widetilde{\Phi}_{n} \neq \widetilde{\Phi}_{n}^{\text {ON }}$, but the polynomials $\widetilde{\Phi}_{n}$ and $\Phi_{n}$ have the same leading coefficient. We have the following relation:

$$
\widetilde{\Phi}_{n}^{\mathrm{ON}}(z)=\sqrt{\frac{c_{0}}{\tilde{c}_{0}} \prod_{j=0}^{n-1} \frac{1-\left|a_{j}\right|^{2}}{1-\left|b_{j}\right|^{2}}} \cdot \widetilde{\Phi}_{n}(z), \quad \tilde{c}_{0}=\frac{1}{2 \pi} \int_{0}^{2 \pi} d \tilde{\sigma}(\varphi) .
$$


In this paper we investigate the question how the polynomials $\left\{\Phi_{n}\right\}_{n \in \mathbb{N}_{0}}$ and $\left\{\widetilde{\Phi}_{n}\right\}_{n \in \mathbb{N}_{0}}$ (resp. their distributions $\sigma$ and $\tilde{\sigma}$ ) are asymptotically related to each other if

$$
\sum_{n=0}^{\infty} \varepsilon_{n}<\infty, \quad \text { where } \varepsilon_{n}:=\left|a_{n}-b_{n}\right|
$$

holds; i.e. the $b_{n}$ 's arise from a perturbation of the $a_{n}$ 's. In particular we are interested in comparative asymptotics such as $\lim _{n \rightarrow \infty}\left(\frac{\Phi_{n}(z)}{\Phi_{n}^{*}(z)}-\frac{\widetilde{\Phi}_{n}(z)}{\widetilde{\Phi}_{n}^{*}(z)}\right), \lim _{n \rightarrow \infty} \frac{\widetilde{\Phi}_{n}^{*}(z)}{\Phi_{n}^{*}(z)}$ etc.

The special and now classical case $a_{n}=0$, i.e. $\left\{\Phi_{n}(z)=z^{n}\right\}_{n \in \mathbb{N}_{0}}$ is the comparative system, has been studied in detail by Geronimus in $[6, \S 26]$ and [4], who obtained asymptotics valid on the whole closed unit disk. Before Geronimus, the large class of distribution functions satisfying the weaker condition (the Szegö-condition)

$$
\sum_{n=0}^{\infty}\left|b_{n}\right|^{2}<\infty
$$

has been investigated by Szegö (cf. $[4,6,22])$. One of the important and well-known results is the equivalence of the following four statements (see e.g. [6, Theorem 21.1]):

(i) The recurrence coefficients $b_{n}$ of $\left\{\widetilde{P}_{n}\right\}_{n \in \mathbb{N}_{0}}$ satisfy (1.13).

(ii) $\lim _{n \rightarrow \infty} \tilde{d}_{n}:=\lim _{n \rightarrow \infty} \prod_{j=0}^{n-1}\left(1-\left|b_{n}\right|^{2}\right)>0$.

(iii) The absolutely continuous part $\tilde{\sigma}^{\prime}$ satisfies Szegö's condition; i.e.

$$
\int_{0}^{2 \pi} \log \tilde{\sigma}^{\prime}(\varphi) d \varphi>-\infty
$$

$$
\lim _{n \rightarrow \infty} \widetilde{\Phi}_{n}^{\mathrm{ON} *}(z)=\frac{1}{D\left(z, \tilde{\sigma}^{\prime}\right)} \quad \text { uniformly on }|z| \leq r<1,
$$

where $D\left(z, \tilde{\sigma}^{\prime}\right)$ is the so-called Szegö-function defined by

$$
D(z, f):=\exp \left\{\frac{1}{4 \pi} \int_{0}^{2 \pi} \frac{e^{i \varphi}+z}{e^{i \varphi}-z} \log f(\varphi) d \varphi\right\}, \quad|z|<1
$$

where $f$ is a nonnegative measurable function that satisfies Szegö's condition $\log f \in L_{1}[0,2 \pi]$. Moreover the radial boundary values $D\left(e^{i \varphi}, f\right):=$ $\lim _{r \rightarrow 1^{-}} D\left(r e^{i \varphi}, f\right)$ exist a.e. on $[0,2 \pi]$ and there holds (cf. [4])

$$
\left|D\left(e^{i \varphi}, f\right)\right|^{2}=f(\varphi) \quad \text { a.e. on }[0,2 \pi]
$$

If one of the above conditions (i)-(iv) is fulfilled (and thus each of them) we further have (with the help of [4, formula (1.12)])

$$
\lim _{n \rightarrow \infty} \widetilde{\Phi}_{n}^{\text {ON }}(z)=0 \quad \text { uniformly on }|z| \leq r<1 .
$$

Comparative asymptotics for distributions $\sigma$ and $\tilde{\sigma}$ not necessarily in the Szegöclass, more precisely for distributions $\sigma, \tilde{\sigma}$ satisfying the condition $\sigma^{\prime}>0$ a.e. on $[0,2 \pi], \tilde{\sigma}$ related to $\sigma$ by $d \tilde{\sigma}=g d \sigma$, where $g \geq 0, Q\left(e^{i \varphi}\right) g(\varphi)$ and $Q\left(e^{i \varphi}\right) / g(\varphi)$ bounded on $[0,2 \pi]$ for a suitable polynomial $Q$, have been obtained by Maté, Nevai 
and Totik $[8,9]$. A typical result in this respect is the following (see [8, Theorem $1])$.

$$
\lim _{n \rightarrow \infty} \frac{\widetilde{\Phi}_{n}^{\mathrm{ON} *}(z)}{\Phi_{n}^{*}(z)}=D\left(z, \frac{\sigma^{\prime}}{\tilde{\sigma}^{\prime}}\right) \quad \text { uniformly on }|z| \leq r<1,
$$

where (uniform) convergence holds also on a subarc $\Gamma$ of $|z|=1$, if $g$ satisfies a Lipschitz condition there; see [9, Theorem 2.3] and also Rakhmanov [21, Theorem $2]$. Note that all these results apply only for such distributions the support of which is $[0,2 \pi]$. By the way, from a result of Geronimus we have (cf. [6, Theorem 19.1])

$$
\operatorname{supp}(\sigma)=[0,2 \pi] \quad \text { if } \quad \lim _{n \rightarrow \infty} a_{n}=0
$$

But let us mention that $\operatorname{supp}(\sigma)=[0,2 \pi]$ does not imply $\lim _{n \rightarrow \infty} a_{n}=0$, as the example in [6, p. 38] shows.

The only exception where asymptotics are given for polynomials orthogonal with respect to a measure whose support is a subset of $(0,2 \pi)$ (note that $\operatorname{supp}(\sigma)$ is a closed set, see e.g. [2, p. 12]), more precisely consists of exactly one interval of the form $[\alpha, 2 \pi-\alpha]$, is the paper of Akhiezer [1]. It is interesting that in this case the structure of the support and the recurrence coefficients are related by the following fact (cf. [3, Theorem $1^{\prime}$, p.206]):

$$
\begin{aligned}
& \lim _{n \rightarrow \infty} a_{n}=a, 0<|a|<1 \text {, implies that there exists } \\
& \text { an } \alpha \in(0,2 \pi) \text { such that }[\alpha, 2 \pi-\alpha] \subseteq \operatorname{supp}(\sigma) \text { and } \\
& \operatorname{supp}(\sigma) \backslash[\beta, 2 \pi-\beta] \text { is finite for every } 0<\beta<\alpha .
\end{aligned}
$$

Let us also mention in this connection (cf. (1.14)) that it follows from [6, Theorem $21.1(\mathrm{IV})]$ that

$$
\left|\Phi_{n}^{*}(z)\right| \underset{n \rightarrow \infty}{\longrightarrow} \infty \text { for }|z|<1 \text { if } \lim _{n \rightarrow \infty} a_{n} \neq 0 \text { or does not exist. }
$$

Hence up to the above-mentioned (classical) cases treated by Szegö, Geronimus, Akhiezer and the special case of a finite perturbation investigated in [14], it is not known how a perturbation of the recurrence coefficients affects the asymptotic behaviour of the perturbed orthogonal polynomials. There are even no (comparative) asymptotics for polynomials orthogonal on a set consisting of several arcs. In this paper we derive under weak assumptions (which are satisfied by polynomials on several arcs, for example) on $\left\{\Phi_{n}\right\}_{n \in \mathbb{N}_{0}}$ and assumption (1.12) comparative asymptotics for $\Phi_{n}^{*}$ and $\widetilde{\Phi}_{n}^{*}$, which hold inside of the unit circle and outside of $\operatorname{supp}(\sigma)$ on $|z|=1$ or, under different assumptions, on compact subsets of the support. In our approach we heavily use associated polynomials and functions of the second kind on the unit circle, studied by the first author in $[13,14]$, which enable us to carry over some ideas of Nevai and Van Assche [11] to the complex case. Naturally if one considers $\lim _{n \rightarrow \infty} \widetilde{\Phi}_{n}^{*} / \Phi_{n}^{*}$ then the behaviour of the comparison system $\left\{\Phi_{n}\right\}_{n \in \mathbb{N}_{0}}$ is of particular interest. For the case of asymptotic periodic recurrence coefficients and the case that $\operatorname{supp}(\sigma)$ consists of several arcs of the unit circle this has been investigated by the authors in $[15,17,18]$.

As we have learned quite recently Golinskii, Nevai and Van Assche are also working on this subject and they got nice results for perturbations of systems satisfying $\lim _{n \rightarrow \infty} a_{n}=a$ (cf. [12]). 
Let us introduce some further notations: The sequence of the so-called polynomials of the second kind of $\left\{P_{n}\right\}_{n \in \mathbb{N}_{0}}$, denoted by $\left\{\Omega_{n}\right\}_{n \in \mathbb{N}_{0}}$, is defined by

$$
\Omega_{n+1}(z)=z \Omega_{n}(z)+\bar{a}_{n} \Omega_{n}^{*}(z), \quad n \in \mathbb{N}_{0}, \Omega_{0}(z)=1,
$$

and the sequence of the $m$ th, $m \in \mathbb{N}_{0}$, associated polynomials $\left\{P_{n}^{(m)}\right\}_{n \in \mathbb{N}_{0}}$ and $\left\{\Omega_{n}^{(m)}\right\}_{n \in \mathbb{N}_{0}}$ by

$$
\begin{aligned}
& P_{n+1}^{(m)}(z)=z P_{n}^{(m)}(z)-\bar{a}_{n+m} P_{n}^{(m) *}(z), \quad n \in \mathbb{N}_{0}, P_{0}^{(m)}(z)=1, \\
& \Omega_{n+1}^{(m)}(z)=z \Omega_{n}^{(m)}(z)+\bar{a}_{n+m} \Omega_{n}^{(m) *}(z), \quad n \in \mathbb{N}_{0}, \Omega_{0}^{(m)}(z)=1 .
\end{aligned}
$$

Again we write $P_{n}$ resp. $\Omega_{n}$ instead of $P_{n}^{(0)}$ resp. $\Omega_{n}^{(0)}$. As in (1.5) we set

$$
\Phi_{n}^{(m)}(z):=\frac{P_{n}^{(m)}(z)}{\sqrt{c_{0} d_{n}^{(m)}}} \quad \text { and } \quad \Psi_{n}^{(m)}(z):=\frac{\Omega_{n}^{(m)}(z)}{\sqrt{c_{0} d_{n}^{(m)}}}, \quad n, m \in \mathbb{N}_{0},
$$

where we choose the norming factor $1 / \sqrt{c_{0} d_{n}^{(m)}}$ for technical reasons. Note that the polynomials $\sqrt{c_{0} / c_{0}^{(m)}} \Phi_{n}^{(m)}$ are orthonormal with respect to the associated distribution function $\sigma^{(m)}$, where $c_{0}^{(m)}=\frac{1}{2 \pi} \int_{0}^{2 \pi} d \sigma^{(m)}(\varphi)$. Let $\widetilde{\Omega}_{n}$ be the polynomial of the second kind of $\widetilde{P}_{n}$. Then we set (compare (1.10))

$$
\widetilde{\Psi}_{n}(z):=\frac{\widetilde{\Omega}_{n}(z)}{\sqrt{c_{0} d_{n}}} .
$$

Now the following statements, which we will need in what follows, hold: Since $\Phi_{n}$ has all zeros in the open unit disk (by (1.2); cf. [6, Theorem 9.1]) we have for all $n \in \mathbb{N}_{0}$

$$
\begin{array}{ll}
\left|\Phi_{n}(z)\right|<\left|\Phi_{n}^{*}(z)\right| & \text { for }|z|<1, \\
\left|\Phi_{n}(z)\right|=\left|\Phi_{n}^{*}(z)\right| & \text { for }|z|=1, \\
\left|\Phi_{n}(z)\right|>\left|\Phi_{n}^{*}(z)\right| & \text { for }|z|>1,
\end{array}
$$

which could also be derived from the relation (cf. $[5,(1.7)]$ )

$$
\sum_{\nu=0}^{n}\left|\Phi_{\nu}(z)\right|^{2}=\frac{\left|\Phi_{n+1}^{*}(z)\right|^{2}-\left|\Phi_{n+1}(z)\right|^{2}}{1-|z|^{2}}, \quad n \in \mathbb{N}_{0}, z \in \mathbb{C} .
$$

Some other useful relations are:

$$
\lim _{n \rightarrow \infty} \frac{\Psi_{n}^{*}(z)}{\Phi_{n}^{*}(z)}=F(z) \quad \text { uniformly for }|z| \leq r<1
$$

(cf. [6, Theorem 13.1]) and

$$
\Phi_{n}^{*}(z) \Psi_{n}(z)+\Phi_{n}(z) \Psi_{n}^{*}(z)=\frac{2}{c_{0}} z^{n} \quad \text { for all } n \in \mathbb{N}_{0}
$$

(cf. $[6,(5.6)])$.

As in $\left[13\right.$, p. 159] let us define the $n$ th, $n \in \mathbb{N}_{0}$, function of the second kind

$$
\begin{aligned}
\mathcal{G}_{n}(z) & =\frac{1}{z^{n}}\left(\Phi_{n}(z) F(z)+\Psi_{n}(z)\right) \\
& =\frac{1}{2 \pi c_{0} z^{n}} \int_{0}^{2 \pi} \frac{e^{i \varphi}+z}{e^{i \varphi}-z} \Phi_{n}\left(e^{i \varphi}\right) d \sigma(\varphi)=2 \sqrt{\frac{d_{n}}{c_{0}}}+O(z), \quad|z|<1,
\end{aligned}
$$


and the $n$th associated function of the second kind

$(1.30)$

$$
\begin{aligned}
\mathcal{H}_{n}(z) & =\frac{1}{z^{n+1}}\left(\Phi_{n}^{*}(z) F(z)-\Psi_{n}^{*}(z)\right) \\
& =\frac{1}{2 \pi c_{0} z} \int_{0}^{2 \pi} \frac{e^{i \varphi}+z}{e^{i \varphi}-z} \overline{\Phi_{n}\left(e^{i \varphi}\right)} d \sigma(\varphi)=2 a_{n} \sqrt{\frac{d_{n}}{c_{0}}}+O(z), \quad|z|<1,
\end{aligned}
$$

where the last equation in (1.29) resp. (1.30) follows from [6, p. 35]. These functions of the second kind investigated by the first author in [13] will play an important role in what follows and have the following properties (see [13, Theorem 2.1 and Lemma 2.2]):

The functions $\mathcal{G}_{n}$ and $\mathcal{H}_{n}$ satisfy a recurrence relation of the form

$$
\begin{aligned}
\sqrt{1-\left|a_{n}\right|^{2}} z \mathcal{H}_{n+1}(z) & =\mathcal{H}_{n}(z)-a_{n} \mathcal{G}_{n}(z), \\
\sqrt{1-\left|a_{n}\right|^{2}} \mathcal{G}_{n+1}(z) & =\mathcal{G}_{n}(z)-\bar{a}_{n} \mathcal{H}_{n}(z),
\end{aligned}
$$

they are analytic on $|z|<1$ and both sequences $\left\{\mathcal{H}_{n}\right\}_{n \in \mathbb{N}_{0}}$ and $\left\{\sqrt{c_{0} d_{n}} \mathcal{G}_{n}\right\}_{n \in \mathbb{N}_{0}}$ converge uniformly on $|z| \leq r<1$, where

$$
\lim _{n \rightarrow \infty} \mathcal{H}_{n}(z)=0 \quad \text { uniformly on }|z| \leq r<1
$$

and where the sequence $\left\{\mathcal{G}_{n}\right\}_{n \in \mathbb{N}_{0}}$ is uniformly bounded on $|z| \leq r<1$. Furthermore, if the finite limit $\lim _{n \rightarrow \infty} 1 / \sqrt{c_{0} d_{n}}$ exists (i.e. if $\left\{\Phi_{n}\right\}_{n \in \mathbb{N}_{0}}$ is in the Szegöclass) then

$$
\lim _{n \rightarrow \infty} \mathcal{G}_{n}(z)=\frac{1}{c_{0}} D\left(z, \sigma^{\prime}\right) \quad \text { uniformly on }|z| \leq r<1 .
$$

Some further useful relations between the $\mathcal{H}_{n}$ 's and $\mathcal{G}_{n}$ 's are:

$$
\left|\mathcal{H}_{n}(z)\right| \leq\left|\mathcal{G}_{n}(z)\right| \text { for }|z|<1
$$

(this can be derived from $[6,(12.13)])$. By the integral representations in (1.29) and (1.30) we have

$$
\left|\mathcal{H}_{n}(z)\right|=\left|\mathcal{G}_{n}(z)\right| \quad \text { for } z \in e^{i \varphi}, \varphi \notin \operatorname{supp}(\sigma)
$$

and by $[13,(2.10)]$ (also directly from (1.29) and (1.30))

$$
\Phi_{n}^{*}(z) \mathcal{G}_{n}(z)-z \Phi_{n}(z) \mathcal{H}_{n}(z)=\frac{2}{c_{0}}
$$

for all $n \in \mathbb{N}_{0}$ and for all $z \in \mathbb{C}$ where $F(z)$ is defined.

In several proofs we will use a discrete version of Gronwall's inequality (see [23, (2.12)]): Suppose $c_{n}$ and $d_{n}, n \in \mathbb{N}_{0}$, are nonnegative real numbers such that

$$
c_{n} \leq A+\sum_{k=0}^{n-1} d_{k} c_{k},
$$

where $A$ is a positive constant; then

$$
c_{n} \leq A \cdot \exp \left(\sum_{k=0}^{n-1} d_{k}\right)
$$


Finally let us mention that, as usual, we call a sequence of (complex) functions $\left\{f_{n}\right\}_{n \in \mathbb{N}_{0}}$ uniformly bounded on a set $\mathcal{M}$, if there exists a constant $K \in \mathbb{R}^{+}$such that

$$
\left|f_{n}(z)\right| \leq K \quad \text { for all } n \in \mathbb{N}_{0}, z \in \mathcal{M}
$$

In this paper we will number the formulas within a proof by $((1)),((2))$, etc. Every proof will start with the number ((1)).

This paper is organized as follows: In Section 2 it is shown how to obtain information on the asymptotic behaviour of orthogonal polynomials with perturbed recurrence coefficients from the asymptotic behaviour of the undisturbed orthogonal polynomials, where the comparison system - the undisturbed orthogonal polynomials - is supposed to satisfy only the conditions (1.2) and (1.4). In Section 3 we study asymptotic properties of the perturbed orthogonal polynomials on $|z|=1$ and show how the orthogonality measures $d \sigma$ and $d \tilde{\sigma}$ are related to each other. Finally we give (under some further assumptions) an explicit expression in terms of $\sigma$ and $\tilde{\sigma}$ for $\lim _{n \rightarrow \infty} \widetilde{\Phi}_{n}^{*}(z) / \Phi_{n}^{*}(z), z \in \mathcal{K}$, where $\mathcal{K}$ is a compact subset of $\{z \in \mathbb{C}:|z| \leq 1\} \backslash\left\{e^{i \varphi}: \varphi \in \operatorname{supp}(\sigma)\right\}$.

\section{AsYmptotic PROPERTIES OF ORTHOGONAL POLYNOMIALS WITH PERTURBED RECURRENCE COEFFICIENTS ON $|z|<1$}

We suppose that the asymptotic behaviour of the polynomials $\left\{\Phi_{n}\right\}_{n \in \mathbb{N}_{0}}$ is known and use these polynomials as a "comparison system" to study asymptotic properties of $\left\{\widetilde{\Phi}_{n}\right\}_{n \in \mathbb{N}_{0}}$.

The following lemma helps to compare $\Phi_{n}$ and $\widetilde{\Phi}_{n}$.

Lemma 2.1. Let $n \in \mathbb{N}_{0}$. The following identities hold:

(a) $\Phi_{n} \widetilde{\Phi}_{n}^{*}-\Phi_{n}^{*} \widetilde{\Phi}_{n}=\sum_{\nu=0}^{n-1} \lambda_{\nu}^{(n)} z^{n-1-\nu}\left\{z\left(\bar{a}_{\nu} b_{\nu}-a_{\nu} \bar{b}_{\nu}\right) \Phi_{\nu}^{*} \widetilde{\Phi}_{\nu}\right.$

$$
\left.+z^{2}\left(a_{\nu}-b_{\nu}\right) \Phi_{\nu} \widetilde{\Phi}_{\nu}-\left(\bar{a}_{\nu}-\bar{b}_{\nu}\right) \Phi_{\nu}^{*} \widetilde{\Phi}_{\nu}^{*}\right\}
$$

where

$$
\lambda_{\nu}^{(n)}=\frac{1}{1-\left|a_{\nu}\right|^{2}} \prod_{j=\nu+1}^{n-1} \frac{1-a_{j} \bar{b}_{j}}{1-\left|a_{j}\right|^{2}}
$$

$$
\begin{aligned}
\widetilde{\Phi}_{n}^{*} \mathcal{G}_{n}-z \widetilde{\Phi}_{n} \mathcal{H}_{n}=\frac{2}{c_{0}}\left[\prod_{j=0}^{n-1} \frac{1-a_{j} \bar{b}_{j}}{1-\left|a_{j}\right|^{2}}\right] & +\sum_{\nu=0}^{n-1} \lambda_{\nu}^{(n)}\left\{z\left(\bar{a}_{\nu} b_{\nu}-a_{\nu} \bar{b}_{\nu}\right) \widetilde{\Phi}_{\nu} \mathcal{H}_{\nu}\right. \\
& \left.+z\left(a_{\nu}-b_{\nu}\right) \widetilde{\Phi}_{\nu} \mathcal{G}_{\nu}-\left(\bar{a}_{\nu}-\bar{b}_{\nu}\right) \widetilde{\Phi}_{\nu}^{*} \mathcal{H}_{\nu}\right\}
\end{aligned}
$$

(c) $\widetilde{\Phi}_{n}=\alpha_{n} \Phi_{n}-\frac{1}{2} \sum_{\nu=0}^{n-1} \lambda_{\nu}^{(n)}\left\{\left(\bar{a}_{\nu} b_{\nu}-a_{\nu} \bar{b}_{\nu}\right) \widetilde{\Phi}_{\nu} \mathcal{A}_{n-\nu}+z\left(a_{\nu}-b_{\nu}\right) \widetilde{\Phi}_{\nu} \mathcal{B}_{n-\nu}\right.$

$$
\left.-\left(\bar{a}_{\nu}-\bar{b}_{\nu}\right) \widetilde{\Phi}_{\nu}^{*} \frac{\mathcal{A}_{n-\nu}}{z}\right\}
$$


where

$$
\begin{aligned}
\mathcal{A}_{n-\nu} & =\Psi_{n-\nu}^{(\nu)}+\Phi_{n-\nu}^{(\nu)} \in \mathbb{P}_{n-\nu}^{\mathbb{C}}, \quad \mathcal{A}_{n-\nu}(0)=0, \\
\mathcal{B}_{n-\nu} & =\Psi_{n-\nu}^{(\nu)}-\Phi_{n-\nu}^{(\nu)} \in \mathbb{P}_{n-\nu-1}^{\mathbb{C}}, \\
\alpha_{n} & =1+\frac{1}{\sqrt{c_{0}}} \sum_{\nu=0}^{n-1} \lambda_{\nu}^{(n)} a_{\nu}\left(\bar{a}_{\nu}-\bar{b}_{\nu}\right) .
\end{aligned}
$$

Proof. Part (a) follows from (1.1), (1.5), (1.8) and (1.10) by straightforward calculation. To prove part (b) we further use (1.29)-(1.31).

(c) Let $n, \nu \in \mathbb{N}_{0}, \nu \leq n$. With the help of $[14,(3.7)]$ it follows:

$$
\begin{aligned}
& \Phi_{n} \Psi_{n-\nu}^{(\nu) *}+\Phi_{n}^{*} \Psi_{n-\nu}^{(\nu)}=z^{n-\nu}\left(\Phi_{\nu}+\Phi_{\nu}^{*}\right), \\
& \Phi_{n} \Phi_{n-\nu}^{(\nu) *}-\Phi_{n}^{*} \Phi_{n-\nu}^{(\nu)}=z^{n-\nu}\left(\Phi_{\nu}-\Phi_{\nu}^{*}\right),
\end{aligned}
$$

and therefore

$$
\begin{aligned}
& 2 z^{n-\nu} \Phi_{\nu}=\mathcal{A}_{n-\nu}^{*} \Phi_{n}+\mathcal{B}_{n-\nu} \Phi_{n}^{*}, \\
& 2 z^{n-\nu} \Phi_{\nu}^{*}=\mathcal{B}_{n-\nu}^{(*)} \Phi_{n}+\mathcal{A}_{n-\nu} \Phi_{n}^{*},
\end{aligned}
$$

where $\mathcal{A}_{n-\nu}, \mathcal{B}_{n-\nu}$ are defined as in the lemma and $\mathcal{A}_{n-\nu}^{*}=\Psi_{n-\nu}^{(\nu) *}+\Phi_{n-\nu}^{(\nu) *}, \mathcal{B}_{n-\nu}^{(*)}=$ $\Psi_{n-\nu}^{(\nu) *}-\Phi_{n-\nu}^{(\nu) *}$ (we use the symbol ${ }^{(*)}$ - which is defined in formally the same way as in (1.3) -instead of *, because the exact degree of $\mathcal{B}_{n-\nu}$ is less than $\left.n-\nu\right)$. If we substitute the expressions $((1))$ in $(2.1)$ we obtain

$$
\Phi_{n} \widetilde{\Phi}_{n}^{*}-\Phi_{n}^{*} \widetilde{\Phi}_{n}=\Phi_{n} \mathcal{C}_{n}-\Phi_{n}^{*} \mathcal{D}_{n}
$$

where

$$
\begin{gathered}
\mathcal{C}_{n}:=\frac{1}{2} \sum_{\nu=0}^{n-1} \lambda_{\nu}^{(n)}\left\{\left(\bar{a}_{\nu} b_{\nu}-a_{\nu} \bar{b}_{\nu}\right) \widetilde{\Phi}_{\nu} \mathcal{B}_{n-\nu}^{(*)}+z\left(a_{\nu}-b_{\nu}\right) \widetilde{\Phi}_{\nu} \mathcal{A}_{n-\nu}^{*}\right. \\
\left.-\left(\bar{a}_{\nu}-\bar{b}_{\nu}\right) \widetilde{\Phi}_{\nu}^{*} \frac{\mathcal{B}_{n-\nu}^{(*)}}{z}\right\} \in \mathbb{P}_{n}^{\mathbb{C}}, \\
\mathcal{D}_{n}:=-\frac{1}{2} \sum_{\nu=0}^{n-1} \lambda_{\nu}^{(n)}\left\{\left(\bar{a}_{\nu} b_{\nu}-a_{\nu} \bar{b}_{\nu}\right) \widetilde{\Phi}_{\nu} \mathcal{A}_{n-\nu}+z\left(a_{\nu}-b_{\nu}\right) \widetilde{\Phi}_{\nu} \mathcal{B}_{n-\nu}\right. \\
\left.-\left(\bar{a}_{\nu}-\bar{b}_{\nu}\right) \widetilde{\Phi}_{\nu}^{*} \frac{\mathcal{A}_{n-\nu}}{z}\right\} \in \mathbb{P}_{n}^{\mathbb{C}} .
\end{gathered}
$$

Let $z_{1}, \ldots, z_{n}$ be the (not necessarily distinct) zeros of $\Phi_{n}$. From the above equation we have

$$
\underbrace{\Phi_{n}^{*}\left(z_{j}\right)}_{\neq 0} \widetilde{\Phi}_{n}\left(z_{j}\right)=\underbrace{\Phi_{n}^{*}\left(z_{j}\right)}_{\neq 0} \mathcal{D}_{n}\left(z_{j}\right), \quad j=1, \ldots, n
$$

(note that $\Phi_{n}^{*}$ has all its zeros in $|z|>1$ by (1.2) and [6, Theorem 9.1]); hence

$$
\widetilde{\Phi}_{n}(z)-\mathcal{D}_{n}(z)=\alpha_{n} \Phi_{n}(z), \quad \alpha_{n} \in \mathbb{C} .
$$


From the definition of $\mathcal{D}_{n}$ and from $((2))$ there follows

$$
\begin{aligned}
\widetilde{\Phi}_{n}=\alpha_{n} \Phi_{n}-\frac{1}{2} \sum_{\nu=0}^{n-1} \lambda_{\nu}^{(n)}\left\{\left(\bar{a}_{\nu} b_{\nu}-a_{\nu} \bar{b}_{\nu}\right) \widetilde{\Phi}_{\nu} \mathcal{A}_{n-\nu}\right. & \\
& \left.+z\left(a_{\nu}-b_{\nu}\right) \widetilde{\Phi}_{\nu} \mathcal{B}_{n-\nu}-\left(\bar{a}_{\nu}-\bar{b}_{\nu}\right) \widetilde{\Phi}_{\nu}^{*} \frac{\mathcal{A}_{n-\nu}}{z}\right\} .
\end{aligned}
$$

Using the fact that $\Phi_{n}(z)=z^{n} / \sqrt{c_{0} d_{n}}+\cdots, \widetilde{\Phi}_{n}(z)=z^{n} / \sqrt{c_{0} d_{n}}+\cdots, \mathcal{A}_{n-\nu}(z)=$ $2 z^{n-\nu} / \sqrt{c_{0} d_{n-\nu}^{(\nu)}}+\cdots, \mathcal{B}_{n-\nu}(z)=2 \bar{a}_{\nu} z^{n-\nu-1} / \sqrt{c_{0} d_{n-\nu}^{(\nu)}}+\cdots$ (by induction) and comparing the leading coefficients in $((3))$ one finds

$$
\alpha_{n}=1+\frac{1}{\sqrt{c_{0}}} \sum_{\nu=0}^{n-1} \lambda_{\nu}^{(n)} a_{\nu}\left(\bar{a}_{\nu}-\bar{b}_{\nu}\right)
$$

This proves our lemma.

The following theorem gives the first asymptotic result.

Theorem 2.1. Let (1.12) be fulfilled, let $M$ be a closed subset of $\{z \in \mathbb{C}:|z|<1\}$ and assume that

$$
\frac{1}{\left|\Phi_{n+1}^{*}(z)\right|^{2}} \sum_{\nu=0}^{n} \varepsilon_{\nu}\left|\Phi_{\nu}^{*}(z)\right|^{2} \leq K \in \mathbb{R}^{+}
$$

for all $n \in \mathbb{N}_{0}$ and $z \in M$, where $\varepsilon_{\nu}$ is defined as in (1.12). Then

$$
\lim _{n \rightarrow \infty}\left(\frac{\Phi_{n}(z)}{\Phi_{n}^{*}(z)}-\frac{\widetilde{\Phi}_{n}(z)}{\widetilde{\Phi}_{n}^{*}(z)}\right)=0 \quad \text { uniformly on } M .
$$

Proof. From (1.4) and (1.12) one obtains the boundedness of the sequence $\left\{\lambda_{\nu}^{(n)}\right\}_{\nu \in \mathbb{N}_{0}}$ uniformly for $n \in \mathbb{N}_{0}$, where $\lambda_{\nu}^{(n)}$ is defined as in Lemma 2.1. Thus there exists a constant $K_{1} \in \mathbb{R}^{+}$such that for all $n, \nu \in \mathbb{N}_{0}$

$$
\left|\lambda_{\nu}^{(n)}\right| \leq K_{1}
$$

Using (1.25a), ((1)) and the fact that $\left|\bar{a}_{\nu} b_{\nu}-a_{\nu} \bar{b}_{\nu}\right| \leq 2\left|a_{\nu}-b_{\nu}\right|$ there follows by relation (2.1) and by Cauchy-Schwarz inequality

$$
\begin{aligned}
\left|\Phi_{n+1} \widetilde{\Phi}_{n+1}^{*}-\Phi_{n+1}^{*} \widetilde{\Phi}_{n+1}\right| & \leq 4 K_{1} \sum_{\nu=0}^{n} \varepsilon_{\nu}|z|^{n-\nu}\left|\Phi_{\nu}^{*}\right|\left|\widetilde{\Phi}_{\nu^{*}}\right| \\
& \leq 4 K_{1} \sqrt{\sum_{\nu=0}^{n} \varepsilon_{\nu}\left|\Phi_{\nu}^{*}\right|^{2} \cdot \sum_{\nu=0}^{n} \varepsilon_{\nu}|z|^{2(n-\nu)}\left|\widetilde{\Phi}_{\nu}^{*}\right|^{2}}
\end{aligned}
$$

and thus

$((2))$

$$
\left|\frac{\Phi_{n+1}}{\Phi_{n+1}^{*}}-\frac{\widetilde{\Phi}_{n+1}}{\widetilde{\Phi}_{n+1}^{*}}\right|^{2} \leq 16 K_{1}^{2}\left[\frac{1}{\left|\Phi_{n+1}^{*}\right|^{2}} \sum_{\nu=0}^{n} \varepsilon_{\nu}\left|\Phi_{\nu}^{*}\right|^{2}\right] \cdot\left[\frac{1}{\left|\widetilde{\Phi}_{n+1}^{*}\right|^{2}} \sum_{\nu=0}^{n} \varepsilon_{\nu}|z|^{2(n-\nu)}\left|\widetilde{\Phi}_{\nu}^{*}\right|^{2}\right]
$$


Besides we get for $|z| \leq r<1$ that there exists a constant $K_{r} \in \mathbb{R}^{+}$such that

$$
\begin{aligned}
\frac{1}{\left|\widetilde{\Phi}_{n+1}^{*}(z)\right|^{2}} \sum_{\nu=0}^{n}|z|^{2(n-\nu)}\left|\widetilde{\Phi}_{\nu}^{*}(z)\right|^{2} & \underset{(1.3)}{=} \frac{\left|\frac{1}{\bar{z}}\right|^{2}}{\left|\widetilde{\Phi}_{n+1}\left(\frac{1}{\bar{z}}\right)\right|^{2}} \sum_{\nu=0}^{n}\left|\widetilde{\Phi}_{\nu}\left(\frac{1}{\bar{z}}\right)\right|^{2} \\
(1.26) & \frac{\frac{\left|\widetilde{\Phi}_{n+1}^{*}\left(\frac{1}{\bar{z}}\right)\right|^{2}}{\left|\widetilde{\Phi}_{n+1}\left(\frac{1}{z}\right)\right|^{2}}-1}{|z|^{2}-1} \leq K_{r},
\end{aligned}
$$

where we have used (1.25c) for the last estimate. From $((3))$ and $\varepsilon_{\nu} \underset{\nu \rightarrow \infty}{\longrightarrow} 0$ there follows by standard techniques

$$
\frac{1}{\left|\widetilde{\Phi}_{n+1}^{*}\right|^{2}} \sum_{\nu=0}^{n} \varepsilon_{\nu}|z|^{2(n-\nu)}\left|\widetilde{\Phi}_{\nu}^{*}\right|^{2} \underset{n \rightarrow \infty}{\longrightarrow} 0 \text { uniformly on } M,
$$

which gives by $((2))$ and (2.4) the assertion.

Remark 2.1. (a) If $\lim _{n \rightarrow \infty} a_{n}=0$ then it's even known (see [8, (3.8), p. 56] or [19, Lemma 6, p. 208]) that

$$
\lim _{n \rightarrow \infty} \frac{\Phi_{n}(z)}{\Phi_{n}^{*}(z)}=0 \quad \text { for }|z|<1 .
$$

Thus Theorem 2.1 gives no new information for this case, since by assumption (1.12) we have $\lim _{n \rightarrow \infty}\left|a_{n}-b_{n}\right|=0$ (and thus $\lim _{n \rightarrow \infty} b_{n}=0$ ). But (2.6) will not hold in general if $\lim _{n \rightarrow \infty} a_{n}$ does not exist or is not equal to zero.

(b) Assumption (2.4) is satisfied by (1.12), if the weak and natural condition (recall (1.21))

$$
\left|\frac{\Phi_{\nu}^{*}(z)}{\Phi_{n}^{*}(z)}\right| \leq \text { const . for all } \nu, n \in \mathbb{N}_{0}, \nu \leq n
$$

holds.

The next theorem shows how the polynomials $\Phi_{n}^{*}$ and $\widetilde{\Phi}_{n}^{*}$ are asymptotically related to each other.

Theorem 2.2. Under the assumptions of Theorem 2.1 the following statements hold:

(a) There exists an analytic function $\Delta$ on $M$ such that

$$
\lim _{n \rightarrow \infty}\left(\left\{\widetilde{\Phi}_{n}^{*}(z) \mathcal{G}_{n}(z)-z \widetilde{\Phi}_{n}(z) \mathcal{H}_{n}(z)\right\}-\Delta(z)\right)=0
$$

uniformly on $M$.

(b)

$$
\lim _{n \rightarrow \infty}\left(\frac{\widetilde{\Phi}_{n}^{*}(z)}{\Phi_{n}^{*}(z)}-\frac{c_{0}}{2} \Delta(z)\right)=0
$$

uniformly on $M$.

Proof. To prove our theorem, we need some preliminary considerations: From the definition of the set $M$ it follows that there exists an $r \in(0,1)$ such that $M \subseteq$ 
$\{z \in \mathbb{C}:|z| \leq r\}$. Thus we get from (1.29)

$$
\begin{aligned}
\left|\Phi_{\nu}^{*}(z) \mathcal{G}_{\nu}(z)\right|=\frac{1}{2 \pi c_{0}}\left|\bar{\Phi}_{\nu}\left(\frac{1}{z}\right) \int_{0}^{2 \pi} \frac{e^{i \varphi}+z}{e^{i \varphi}-z} \Phi_{\nu}\left(e^{i \varphi}\right) d \sigma(\varphi)\right| \\
\leq \frac{1}{2 \pi c_{0}}\left\{\frac{1+r}{1-r} \int_{0}^{2 \pi} \overline{\Phi_{\nu}\left(e^{i \varphi}\right)} \Phi_{\nu}\left(e^{i \varphi}\right) d \sigma(\varphi)\right. \\
\left.+\left|\int_{0}^{2 \pi} \frac{\frac{1}{e^{i \varphi}}+\frac{1}{z}}{\frac{1}{e^{i \varphi}}-\frac{1}{z}}\left(\bar{\Phi}_{\nu}\left(\frac{1}{e^{i \varphi}}\right)-\bar{\Phi}_{\nu}\left(\frac{1}{z}\right)\right) \Phi_{\nu}\left(e^{i \varphi}\right) d \sigma(\varphi)\right|\right\}
\end{aligned}
$$

Since $\Phi_{\nu}$ is orthonormal with respect to $\sigma$ the first integral at the right-hand side of the above inequality is equal to $2 \pi$. Because of

$$
\frac{\frac{1}{e^{i \varphi}}+\frac{1}{z}}{\frac{1}{e^{1 \varphi}}-\frac{1}{z}}\left(\bar{\Phi}_{\nu}\left(\frac{1}{e^{i \varphi}}\right)-\bar{\Phi}_{\nu}\left(\frac{1}{z}\right)\right)=\frac{1}{\sqrt{c_{0} d_{\nu}}} e^{-i \nu \varphi}+\text { terms of lower orders of } e^{-i \varphi}
$$

and the orthogonality-property of $\Phi_{\nu}$ the second integral is equal to $2 \pi$, too. Thus the sequence $\left\{\Phi_{\nu}^{*} \mathcal{G}_{\nu}\right\}_{\nu \in \mathbb{N}_{0}}$ is uniformly bounded on $M$; i.e. for all $\nu \in \mathbb{N}_{0}$

$$
\left|\Phi_{\nu}^{*}(z) \mathcal{G}_{\nu}(z)\right| \leq K_{1} \in \mathbb{R}^{+} \quad \text { on } M .
$$

Now we obtain from (1.25a) and (1.34) for $z \in M$

$$
\left.\begin{array}{r}
\left|\widetilde{\Phi}_{\nu}(z) \mathcal{H}_{\nu}(z)\right| \\
\left|\widetilde{\Phi}_{\nu}^{*}(z) \mathcal{H}_{\nu}(z)\right| \\
\left|\widetilde{\Phi}_{\nu}(z) \mathcal{G}_{\nu}(z)\right|
\end{array}\right\} \leq\left|\widetilde{\Phi}_{\nu}^{*}(z) \mathcal{G}_{\nu}(z)\right|=\left|\frac{\widetilde{\Phi}_{\nu}^{*}(z)}{\Phi_{\nu}^{*}(z)}\right|\left|\Phi_{\nu}^{*}(z) \mathcal{G}_{\nu}(z)\right| \underset{((2))}{\leq} K_{1}\left|\frac{\widetilde{\Phi}_{\nu}^{*}(z)}{\Phi_{\nu}^{*}(z)}\right|
$$

Since $\Phi_{n}^{*}(z) \neq 0$ and $\widetilde{\Phi}_{n}^{*}(z) \neq 0$ for $|z| \leq 1$ (see e.g. [6, Theorem 9.1]) we can write

$$
\widetilde{\Phi}_{n}^{*}(z) \mathcal{G}_{n}(z)-z \widetilde{\Phi}_{n}(z) \mathcal{H}_{n}(z)=\frac{\widetilde{\Phi}_{n}^{*}(z)}{\Phi_{n}^{*}(z)} \cdot v_{n}(z),
$$

where

$$
v_{n}(z):=\Phi_{n}^{*}(z) \mathcal{G}_{n}(z)-z \frac{\widetilde{\Phi}_{n}(z)}{\widetilde{\Phi}_{n}^{*}(z)} \Phi_{n}^{*}(z) \mathcal{H}_{n}(z) .
$$

With the help of $((2))$ and (1.12) we obtain from $((3))$ and (2.2) that for all $z \in M$ (compare the proof of Theorem 2.1)

$$
\left|\frac{\widetilde{\Phi}_{n}^{*}(z)}{\Phi_{n}^{*}(z)}\right| \cdot\left|v_{n}(z)\right| \leq K_{2}+K_{3} \sum_{\nu=0}^{n-1}\left|a_{\nu}-b_{\nu}\right| \cdot\left|\frac{\widetilde{\Phi}_{\nu}^{*}(z)}{\Phi_{\nu}^{*}(z)}\right|, \quad K_{2}, K_{3} \in \mathbb{R}^{+} .
$$

From the definition of the function $v_{n}$ and the uniform boundedness of $\Phi_{n}^{*} \mathcal{H}_{n}$ on $M$ (note ((1)) and (1.34)) we have by Theorem 2.1 and (1.36)

$$
\begin{aligned}
v_{n}(z)= & \Phi_{n}^{*}(z) \mathcal{G}_{n}(z)-z \Phi_{n}(z) \mathcal{H}_{n}(z) \\
& +z\left(\frac{\Phi_{n}(z)}{\Phi_{n}^{*}(z)}-\frac{\widetilde{\Phi}_{n}(z)}{\widetilde{\Phi}_{n}^{*}(z)}\right) \Phi_{n}^{*}(z) \mathcal{H}_{n}(z) \underset{n \rightarrow \infty}{\longrightarrow} \frac{2}{c_{0}}
\end{aligned}
$$

uniformly on $M$. Using the fact (cf. [5, (1.12')])

$$
\left|\Phi_{n}^{*}(z)\right| \geq \sqrt{c_{0}\left(1-|z|^{2}\right)} \text { for all } n \in \mathbb{N}_{0},|z|<1,
$$


we get the uniform boundedness of $\left\{\widetilde{\Phi}_{n}^{*}(z) / \Phi_{n}^{*}(z)\right\}_{n \in \mathbb{N}_{0}}$ on $M$ by $((4)),((5)),(1.12)$ and Gronwall's inequality (1.37), and thus by $((2))$ the uniform boundedness of

$$
\widetilde{\Phi}_{\nu}(z) \mathcal{H}_{\nu}(z), \quad \widetilde{\Phi}_{\nu}^{*}(z) \mathcal{H}_{\nu}(z), \quad \widetilde{\Phi}_{\nu}(z) \mathcal{G}_{\nu}(z)
$$

on $M$. Now the assertion (2.7) follows from (2.2) (note (1.12)) and the assertion (2.8) from (2.7), ((3)) and ((5)).

In Section 3 we will show that under not very restrictive assumptions on $\sigma$ the limit relation (2.7) also holds a.e. on $|z|=1$ and (2.8) for $z=e^{i \varphi}, \varphi \notin \operatorname{supp}(\sigma)$. For (2.8) further compare (1.18) and note that it follows from (2.7) and (1.14), (1.17), (1.32), (1.33) that under the assumptions of Theorem 2.1

$$
\frac{c_{0} \beta}{2} \Delta(z)=D\left(z, \frac{\sigma^{\prime}}{\tilde{\sigma}^{\prime}}\right)
$$

if $\sigma$ is from the Szegö-class, where $\beta \in \mathbb{R} \backslash\{0\}$ (compare (3.13) in Section 3). Thus for the special case of Szegö-class we obtain the representation (1.18) by (2.8).

With the help of the next lemma it follows that Theorem 2.2(b) gives the right asymptotic behaviour.

Lemma 2.2. Let the assumptions of Theorem 2.1 be fulfilled. Then the analytic function $\Delta$, given by (2.7), satisfies $\Delta(z) \neq 0$ for $z \in M$.

Proof. If we denote

$$
\Delta_{n}(z):=\widetilde{\Phi}_{n}^{*}(z) \mathcal{G}_{n}(z)-z \widetilde{\Phi}_{n}(z) \mathcal{H}_{n}(z)
$$

we obtain from $(2.2)$

$$
\begin{array}{r}
\Delta_{n+1}-\left(\frac{1-a_{n} \bar{b}_{n}}{1-\left|a_{n}\right|^{2}}\right) \Delta_{n}=\frac{1}{1-\left|a_{n}\right|^{2}}\left\{z\left(\bar{a}_{n} b_{n}-a_{n} \bar{b}_{n}\right) \widetilde{\Phi}_{n} \mathcal{H}_{n}\right. \\
\left.-\left(\bar{a}_{n}-\bar{b}_{n}\right) \widetilde{\Phi}_{n}^{*} \mathcal{H}_{n}+z\left(a_{n}-b_{n}\right) \widetilde{\Phi}_{n} \mathcal{G}_{n}\right\}
\end{array}
$$

i.e.

$((1))$

$$
\begin{aligned}
& \left(\frac{1-a_{n} \bar{b}_{n}}{1-\left|a_{n}\right|^{2}}\right) \Delta_{n} \\
& \quad=\Delta_{n+1}\left[1-\frac{1}{1-\left|a_{n}\right|^{2}}\left\{z\left(\bar{a}_{n} b_{n}-a_{n} \bar{b}_{n}\right) \widetilde{\Phi}_{n} \frac{\mathcal{H}_{n}}{\Delta_{n+1}}\right.\right. \\
& \left.\left.\quad-\left(\bar{a}_{n}-\bar{b}_{n}\right) \widetilde{\Phi}_{n}^{*} \frac{\mathcal{H}_{n}}{\Delta_{n+1}}+z\left(a_{n}-b_{n}\right) \widetilde{\Phi}_{n} \frac{\mathcal{G}_{n}}{\Delta_{n+1}}\right\}\right] .
\end{aligned}
$$

Let $z \in M$ be fixed. To prove the assertion of the lemma we need some estimates of the expressions $\left|\widetilde{\Phi}_{n} \mathcal{H}_{n} / \Delta_{n+1}\right|,\left|\widetilde{\Phi}_{n}^{*} \mathcal{H}_{n} / \Delta_{n+1}\right|$ and $\left|\widetilde{\Phi}_{n} \mathcal{G}_{n} / \Delta_{n+1}\right|$. First we recall that these expressions are all bounded by $\left|\widetilde{\Phi}_{n}^{*} \mathcal{G}_{n} / \Delta_{n+1}\right|$ by (1.25a) and (1.34).

As we have shown in the proof of Theorem 2.2

$$
\Delta_{n+1}(z)=\frac{\widetilde{\Phi}_{n+1}^{*}(z)}{\Phi_{n+1}^{*}(z)} \cdot v_{n+1}(z),
$$


where $v_{n+1}(z) \underset{n \rightarrow \infty}{\longrightarrow} 2 / c_{0}>0$, we get for $n \geq n_{0}$ and $n_{0}$ sufficiently large

$$
\left|\widetilde{\Phi}_{n}^{*}(z) \frac{\mathcal{G}_{n}(z)}{\Delta_{n+1}(z)}\right| \leq K_{1}\left|\frac{\widetilde{\Phi}_{n}^{*}(z)}{\widetilde{\Phi}_{n+1}^{*}(z)}\right| \cdot\left|\Phi_{n}^{*}(z) \mathcal{G}_{n}(z)\right|, \quad K_{1} \in \mathbb{R}^{+},
$$

where the above inequality can be derived by using (1.1), (1.2), (1.4) and (1.25a). In the proof of Theorem 2.2 we have also shown the boundedness of the sequence $\left\{\Phi_{n}^{*}(z) \mathcal{G}_{n}(z)\right\}_{n \in \mathbb{N}_{0}}$. Since

$$
\left|\frac{\widetilde{\Phi}_{n}^{*}(z)}{\widetilde{\Phi}_{n+1}^{*}(z)}\right|=\frac{\sqrt{1-\left|b_{n}\right|^{2}}}{\left|1-z b_{n} \widetilde{\Phi}_{n}(z) / \widetilde{\Phi}_{n}^{*}(z)\right|}
$$

(by (1.8) and (1.10)) and

$$
\left|1-z b_{n} \frac{\widetilde{\Phi}_{n}(z)}{\widetilde{\Phi}_{n}^{*}(z)}\right| \geq \delta>0 \quad \text { for all } n \in \mathbb{N}_{0}
$$

(recall $|z|<1$ fixed, (1.4), (1.9) and (1.25a)), the sequence $\left\{\widetilde{\Phi}_{n}^{*}(z) / \widetilde{\Phi}_{n+1}^{*}(z)\right\}_{n \in \mathbb{N}_{0}}$ is bounded, and consequently by $((3))$ the sequence $\left\{\widetilde{\Phi}_{n}^{*}(z) \mathcal{G}_{n}(z) / \Delta_{n+1}(z)\right\}_{n \in \mathbb{N}_{0}}$ is bounded, too. Thus, by the above considerations, there exists a constant $K_{2} \in \mathbb{R}^{+}$ such that for all $n \geq n_{0}$ (recall (1.4))

$$
\frac{1}{1-\left|a_{n}\right|^{2}}\left(2\left|z \widetilde{\Phi}_{n}(z) \frac{\mathcal{H}_{n}(z)}{\Delta_{n+1}(z)}\right|+\left|\widetilde{\Phi}_{n}^{*}(z) \frac{\mathcal{H}_{n}(z)}{\Delta_{n+1}(z)}\right|+\left|z \widetilde{\Phi}_{n}(z) \frac{\mathcal{G}_{n}(z)}{\Delta_{n+1}(z)}\right|\right) \leq K_{2},
$$

i.e. by $((1))$

$$
\begin{aligned}
\underbrace{\left|\frac{1-a_{n} \bar{b}_{n}}{1-\left|a_{n}\right|^{2} \mid}\right| \Delta_{n}(z) \mid}_{\neq 0} & \leq\left|\Delta_{n+1}(z)\right|\left(1+K_{2}\left|a_{n}-b_{n}\right|\right) \\
& \leq\left|\Delta_{n+1}(z)\right| \exp \left(K_{2}\left|a_{n}-b_{n}\right|\right) \\
& \leq \cdots \leq\left|\Delta_{n+m}(z)\right| \exp \left(K_{2} \sum_{\nu=n}^{n+m-1}\left|a_{\nu}-b_{\nu}\right|\right), \quad m \in \mathbb{N}
\end{aligned}
$$

If $m$ tends to $\infty$ the right-hand side converges to $|\Delta(z)| \exp \left(K_{2} \sum_{\nu=n}^{\infty}\left|a_{\nu}-b_{\nu}\right|\right)<\infty$ (recall (1.12)). Thus $\Delta(z)=0$ implies for $n \geq n_{0}$

$$
0=\Delta_{n}(z)=\frac{\widetilde{\Phi}_{n}^{*}(z)}{\Phi_{n}^{*}(z)} \cdot v_{n}(z)
$$

i.e. since $v_{n}(z) \neq 0$ (note $v_{n}(z) \underset{n \rightarrow \infty}{\longrightarrow} 2 / c_{0}$ and $n$ sufficiently large) and $\left|\Phi_{n}^{*}(z)\right|<$ $\infty$ we have $\widetilde{\Phi}_{n}^{*}(z)=0$. This contradicts $\widetilde{\Phi}_{n}^{*}(y) \neq 0$ for $|y| \leq 1$ (compare [6, Theorem 9.1]) and proves the lemma.

\section{Asymptotics on $|z|=1$}

Let $\mathcal{N}$ be a compact subset of $[0,2 \pi]$. In this section we will study under which conditions the asymptotic statements of Section 2 hold true also on subarcs

$$
\Gamma_{\mathcal{N}}:=\left\{e^{i \varphi}: \varphi \in \mathcal{N}\right\}
$$

of $|z|=1$. As one expects, we will have to distinguish between the case that $\mathcal{N} \subset \operatorname{supp}(\sigma)$ resp. $\mathcal{N} \cap \operatorname{supp}(\sigma)=\varnothing$ (compare the following Lemma 3.1 and 
the Theorems 3.2 and 3.3). Then at the end of this section we show how the orthogonality measures $d \sigma$ and $d \tilde{\sigma}$ are related to each other.

In what follows we suppose that the radial boundary values

$$
\left\{\begin{array}{l}
F\left(e^{i \psi}\right):=\lim _{r \rightarrow 1^{-}} F\left(r e^{i \psi}\right) \text { exist for all } \psi \in \mathcal{N}, \\
\text { and } F \text { is bounded on } \Gamma_{\mathcal{N}} .
\end{array}\right.
$$

The second assumption is fulfilled, if for example the convergence in (3.1) is uniform on $\Gamma_{\mathcal{N}}$ (compare Lemma 3.1 below). Thus we are able to define in the sense of (3.1) (see (1.29) resp. (1.30))

$$
\begin{aligned}
\mathcal{G}_{n}\left(e^{i \psi}\right) & :=e^{-i n \psi}\left(\Phi_{n}\left(e^{i \psi}\right) F\left(e^{i \psi}\right)+\Psi_{n}\left(e^{i \psi}\right)\right), \\
\mathcal{H}_{n}\left(e^{i \psi}\right) & :=e^{-i(n+1) \psi}\left(\Phi_{n}^{*}\left(e^{i \psi}\right) F\left(e^{i \psi}\right)-\Psi_{n}^{*}\left(e^{i \psi}\right)\right), \quad n \in \mathbb{N}_{0}, \psi \in \mathcal{N} .
\end{aligned}
$$

Note, if $\psi \notin \operatorname{supp}(\sigma)$ we have by (1.7), (1.29) and (1.30)

$$
\begin{aligned}
F\left(e^{i \psi}\right) & =\frac{1}{2 \pi c_{0}} \int_{\operatorname{supp}(\sigma)} \frac{e^{i \varphi}+e^{i \psi}}{e^{i \varphi}-e^{i \psi}} d \sigma(\varphi), \\
\mathcal{G}_{n}\left(e^{i \psi}\right) & =\frac{1}{2 \pi c_{0} e^{i n \psi}} \int_{\operatorname{supp}(\sigma)} \frac{e^{i \varphi}+e^{i \psi}}{e^{i \varphi}-e^{i \psi}} \Phi_{n}\left(e^{i \varphi}\right) d \sigma(\varphi), \\
\mathcal{H}_{n}\left(e^{i \psi}\right) & =\frac{1}{2 \pi c_{0} e^{i \psi}} \int_{\operatorname{supp}(\sigma)} \frac{e^{i \varphi}+e^{i \psi}}{e^{i \varphi}-e^{i \psi}} \overline{\Phi_{n}\left(e^{i \varphi}\right)} d \sigma(\varphi) .
\end{aligned}
$$

The following (useful) lemma shows that the situation for $\mathcal{N} \cap \operatorname{supp}(\sigma)=\varnothing$ is pleasant in general.

Lemma 3.1. Suppose $\mathcal{N} \subset \stackrel{\circ}{\mathcal{M}}, \mathcal{M}$ compact subset of $[0,2 \pi], \mathcal{M} \cap \operatorname{supp}(\sigma)=\varnothing$ (i.e. $\mathcal{N}$ has a positive distance from $\operatorname{supp}(\sigma))$ and $\operatorname{let} \mathcal{U}_{\delta}(\mathcal{N}):=\left\{r e^{i \psi}: r \in[\delta, 1], \psi \in \mathcal{N}\right\}$, $\delta \in(0,1)$. Then the following statements hold:

(a) $\lim _{|z| \rightarrow 1^{-}} F(z)$ exists uniformly on $\mathcal{U}_{\delta}(\mathcal{N})$ (i.e. assumption (3.1) is fulfilled).

$$
\left.\mathcal{H}_{n}(z) \underset{n \rightarrow \infty}{\longrightarrow} 0 \quad \text { uniformly on } \mathcal{U}_{\delta}(\mathcal{N}) \quad \text { (compare }(1.32)\right)
$$

$$
\frac{\Psi_{n}^{*}(z)}{\Phi_{n}^{*}(z)} \underset{n \rightarrow \infty}{\longrightarrow} F(z) \quad \text { uniformly on } \mathcal{U}_{\delta}(\mathcal{N}) \quad(\text { compare }(1.27))
$$

$$
\left|\Phi_{n}^{*}\left(e^{i \psi}\right)\right| \underset{n \rightarrow \infty}{\longrightarrow} \infty \quad \text { uniformly on } \mathcal{N} \quad(\text { compare }(1.21)) .
$$

Proof. The assertion (a) follows from the continuity of $F$ on $\mathcal{U}_{\delta}(\mathcal{N})$ (compare (3.3a)).

(b) Let $z_{1}, z_{2} \in \mathcal{U}_{\delta}(\mathcal{N})$. Then we obtain from (1.30) and (3.3c)

$$
\begin{aligned}
\left|\mathcal{H}_{n}\left(z_{1}\right)-\mathcal{H}_{n}\left(z_{2}\right)\right| & =\frac{1}{2 \pi c_{0}}\left|\int_{\operatorname{supp}(\sigma)}\left[\frac{1}{z_{1}} \frac{e^{i \varphi}+z_{1}}{e^{i \varphi}-z_{1}}-\frac{1}{z_{2}} \frac{e^{i \varphi}+z_{2}}{e^{i \varphi}-z_{2}}\right] \overline{\Phi_{n}\left(e^{i \varphi}\right)} d \sigma(\varphi)\right| \\
& \leq \frac{1}{2 \pi c_{0}} g\left(\left|z_{1}-z_{2}\right|\right) \int_{\operatorname{supp}(\sigma)}\left|\Phi_{n}\left(e^{i \varphi}\right)\right| d \sigma(\varphi)
\end{aligned}
$$


where

$$
\begin{array}{r}
g(\varepsilon):=\sup \left\{\left|\frac{1}{y_{1}} \frac{e^{i \varphi}+y_{1}}{e^{i \varphi}-y_{1}}-\frac{1}{y_{2}} \frac{e^{i \varphi}+y_{2}}{e^{i \varphi}-y_{2}}\right|: \varphi \in \operatorname{supp}(\sigma)\right. \\
\left.y_{1}, y_{2} \in \mathcal{U}_{\delta}(\mathcal{N}),\left|y_{1}-y_{2}\right| \leq \varepsilon\right\} .
\end{array}
$$

Since $\sigma$ is a distribution function, we have by Cauchy-Schwarz inequality and by the orthogonality property of $\Phi_{n}$

$\int_{\operatorname{supp}(\sigma)} 1 \cdot\left|\Phi_{n}\left(e^{i \varphi}\right)\right| d \sigma(\varphi) \leq \sqrt{\int_{\operatorname{supp}(\sigma)} d \sigma(\varphi)} \sqrt{\int_{\operatorname{supp}(\sigma)}\left|\Phi_{n}\left(e^{i \varphi}\right)\right|^{2} d \sigma(\varphi)}=2 \pi \sqrt{c_{0}}$

i.e.

((1)) $\quad\left|\mathcal{H}_{n}\left(z_{1}\right)-\mathcal{H}_{n}\left(z_{2}\right)\right| \leq \frac{1}{\sqrt{c_{0}}} g\left(\left|z_{1}-z_{2}\right|\right) \quad$ (independent of $n$ ).

Since $\frac{1}{z} \frac{e^{i \varphi}+z}{e^{i \varphi}-z}$ is a uniform continuous function on $\operatorname{supp}(\sigma) \times \mathcal{U}_{\delta}(\mathcal{N})$ (recall that $\mathcal{N}$ has a positive distance from $\operatorname{supp}(\sigma)$ and $|z| \geq \delta>0)$, we obtain

$$
g(\varepsilon) \underset{\varepsilon \rightarrow 0}{\longrightarrow} 0 .
$$

Now (3.4) follows from (1.32), ((1)) and ((2))

(c) In view of (3.4) there exists an $n_{0} \in \mathbb{N}_{0}$ such that for all $n \geq n_{0}$.

$$
\left|z \mathcal{H}_{n}(z)\right|=\left|\frac{\Phi_{n}^{*}(z)}{z^{n}} F(z)-\frac{\Psi_{n}^{*}(z)}{z^{n}}\right|<\frac{1}{2 \sqrt{c_{0}}} \quad \text { on } \mathcal{U}_{\delta}(\mathcal{N})
$$

Let

$$
K:=\max \left(1, \sup _{z \in \mathcal{U}_{\delta}(\mathcal{N})}|F(z)|\right)<\infty
$$

(note (1.7) resp. (3.3a)); then there holds

$$
\left|\frac{\Phi_{n}^{*}(z)}{z^{n}}\right| \geq \frac{1}{2 K \sqrt{c_{0}}} \quad \text { for all } n \geq n_{0} \text { and } z \in \mathcal{U}_{\delta}(\mathcal{N}) .
$$

Indeed, suppose the opposite, i.e.

$$
\left|\frac{\Phi_{n_{1}}^{*}\left(z_{1}\right)}{z_{1}^{n_{1}}}\right|<\frac{1}{2 K \sqrt{c_{0}}} \quad \text { for a fixed } n_{1} \geq n_{0}, z_{1} \in \mathcal{U}_{\delta}(\mathcal{N})
$$

By $((3))$ there follows

$$
\left|\frac{\Psi_{n_{1}}^{*}\left(z_{1}\right)}{z_{1}^{n_{1}}}\right|<\frac{1}{\sqrt{c_{0}}} .
$$

From $((5)),((6))$ and $(1.25 \mathrm{a})$ we obtain

$$
\left|\Phi_{n_{1}}^{*}\left(z_{1}\right) \Psi_{n_{1}}\left(z_{1}\right)+\Phi_{n_{1}}\left(z_{1}\right) \Psi_{n_{1}}^{*}\left(z_{1}\right)\right| \leq 2\left|\Phi_{n_{1}}^{*}\left(z_{1}\right)\right|\left|\Psi_{n_{1}}^{*}\left(z_{1}\right)\right|<\frac{1}{c_{0}}\left|z_{1}\right|^{n_{1}},
$$

which contradicts (1.28); thus we have shown $((4))$. Now there holds by $(3.4)$ uniformly on $\mathcal{U}_{\delta}(\mathcal{N})$

$$
z \mathcal{H}_{n}(z)=\frac{\Phi_{n}^{*}(z)}{z^{n}}\left(F(z)-\frac{\Psi_{n}^{*}(z)}{\Phi_{n}^{*}(z)}\right) \underset{n \rightarrow \infty}{\longrightarrow} 0,
$$

such that the assertion (3.5) follows from $((4))$ 
(d) From (3.3a)

$$
\operatorname{Re} F\left(e^{i \psi}\right)=0, \quad \psi \in \mathcal{N} .
$$

Now from (3.5) and (1.28) one obtains

$$
\begin{aligned}
\frac{2}{c_{0}\left|\Phi_{n}\left(e^{i \psi}\right)\right|^{2}} & =\frac{\Psi_{n}^{*}\left(e^{i \psi}\right)}{\Phi_{n}^{*}\left(e^{i \psi}\right)}+\overline{\left[\frac{\Psi_{n}^{*}\left(e^{i \psi}\right)}{\Phi_{n}^{*}\left(e^{i \psi}\right)}\right]} \underset{n \rightarrow \infty}{\longrightarrow} F\left(e^{i \psi}\right)+\overline{F\left(e^{i \psi}\right)} \\
& =2 \operatorname{Re} F\left(e^{i \psi}\right)=0
\end{aligned}
$$

uniformly on $\mathcal{N}$, which yields (3.6).

Let us note that in general the statements in Lemma 3.1 will not hold on $\Gamma_{\mathcal{N}}$, if $\mathcal{N} \subset \operatorname{supp}(\sigma)$. For instance, when we studied asymptotic properties of orthogonal polynomials with periodic recurrence coefficients we have shown (see [17]) that (3.4), (3.5) and (3.6) are not valid for any $e^{i \psi}, \psi \in \operatorname{Int}(\operatorname{supp}(\sigma))$.

Next let us show that on the unit circle boundedness of the undisturbed orthonormal polynomials implies boundedness of the disturbed polynomials.

Proposition 3.1. Assume that the polynomials $\Phi_{n}$ and $\Psi_{n}$ are uniformly bounded on $\mathcal{M} \subseteq\{z \in \mathbb{C}:|z|=1\}$ and that (1.12) holds. Then the polynomials $\widetilde{\Phi}_{n}$ and $\widetilde{\Psi}_{n}$ are uniformly bounded on $\mathcal{M}$, too.

Proof. From (1.4) and (1.12) one obtains the boundedness of the sequences $\left\{\lambda_{\nu}^{(n)}\right\}_{\nu \in \mathbb{N}_{0}}$ and $\left\{\alpha_{\nu}\right\}_{\nu \in \mathbb{N}_{0}}$ uniformly for $n \in \mathbb{N}_{0}$, where $\lambda_{\nu}^{(n)}$ and $\alpha_{n}$ are defined as in Lemma 2.1. By (1.25b) and

$$
\begin{aligned}
& 2 \Phi_{n-\nu}^{(\nu)}=\frac{\sqrt{c_{0}}}{z^{\nu}}\left[\Phi_{n}\left(\Psi_{\nu}+\Psi_{\nu}^{*}\right)-\Psi_{n}\left(\Phi_{\nu}-\Phi_{\nu}^{*}\right)\right] \\
& 2 \Psi_{n-\nu}^{(\nu)}=\frac{\sqrt{c_{0}}}{z^{\nu}}\left[\Psi_{n}\left(\Phi_{\nu}+\Phi_{\nu}^{*}\right)-\Phi_{n}\left(\Psi_{\nu}-\Psi_{\nu}^{*}\right)\right]
\end{aligned}
$$

(these equations can be derived from [14, Corollary 3.1]) the polynomials $\Phi_{n-\nu}^{(\nu)}$ and $\Psi_{n-\nu}^{(\nu)}$, and therefore the polynomials $\mathcal{A}_{n-\nu}$ and $\mathcal{B}_{n-\nu}$, are uniformly bounded for $n, \nu \in \mathbb{N}_{0}$ on $\mathcal{M}$. Thus we see from (2.3) (by using again (1.25b) and the fact that $\left.\left|\bar{a}_{\nu} b_{\nu}-a_{\nu} \bar{b}_{\nu}\right| \leq 2\left|a_{\nu}-b_{\nu}\right|\right)$ that there exist real, positive constants $K_{1}$ and $K_{2}$ such that uniformly on $\mathcal{M}$

$$
\left|\widetilde{\Phi}_{n}(z)\right| \leq K_{1}+K_{2} \sum_{\nu=0}^{n-1}\left|a_{\nu}-b_{\nu}\right| \cdot\left|\widetilde{\Phi}_{\nu}(z)\right| .
$$

Using (1.37) we have

$$
\left|\widetilde{\Phi}_{n}(z)\right| \leq K_{1} \cdot \exp \left(K_{2} \sum_{\nu=0}^{\infty}\left|a_{\nu}-b_{\nu}\right|\right) \underset{(1.12)}{<} \infty
$$

for all $n \in \mathbb{N}_{0}$ uniformly on $\mathcal{M}$. By changing the roles of $a_{n}$ and $-a_{n}$ resp. of $b_{n}$ and $-b_{n}$ we get the uniform boundedness of $\left\{\widetilde{\Psi}_{n}\right\}_{n \in \mathbb{N}_{0}}$ on $\mathcal{M}$ (compare (1.1) and (1.22)).

To prove the main results of this section we need the following.

Lemma 3.2. Let $\mathcal{N}$ be a compact subset of $[0,2 \pi]$. 
(a) Suppose $\mathcal{N} \subset \stackrel{\circ}{\mathcal{M}}, \mathcal{M}$ compact subset of $[0,2 \pi]$ and $\mathcal{M} \cap \operatorname{supp}(\sigma)=\varnothing$. Further suppose that uniformly on $\Gamma_{\mathcal{M}}$

$$
0<\lambda_{1} \leq\left|\frac{\mathcal{G}_{n}\left(e^{i \psi}\right)+z \mathcal{H}_{n}\left(e^{i \psi}\right)}{\mathcal{G}_{n}\left(e^{i \psi}\right)-z \mathcal{H}_{n}\left(e^{i \psi}\right)}\right| \leq \lambda_{2}<\infty \quad \text { for all } n \in \mathbb{N}_{0} .
$$

Then there exists a constant $K \in \mathbb{R}^{+}$such that uniformly on $\Gamma_{\mathcal{N}}$

$$
\left|\widetilde{\Phi}_{n}\left(e^{i \psi}\right) \mathcal{G}_{n}\left(e^{i \psi}\right)\right| \leq K \cdot \exp \left(K \sum_{\nu=0}^{n-1}\left|a_{\nu}-b_{\nu}\right|\right) \quad \text { for all } n \in \mathbb{N}_{0} .
$$

(b) Let $\mathcal{N} \subseteq \operatorname{supp}(\sigma)$. Suppose that (1.12) and (3.1) are fulfilled and that the polynomials $\Phi_{n}, \Psi_{n}$ are uniformly bounded on $\Gamma_{\mathcal{N}}$. Then the sequence $\left\{\widetilde{\Phi}_{n}(z) \mathcal{G}_{n}(z)\right\}_{n \in \mathbb{N}_{0}}$ is uniformly bounded on $\Gamma_{\mathcal{N}}$, too.

Proof. (a) Let $d \sigma^{(\nu)}$ denote the orthogonality measure of the $\nu$ th, $\nu \in \mathbb{N}_{0}$, associated polynomials $\left\{\Phi_{n}^{(\nu)}\right\}_{n \in \mathbb{N}_{0}}$, defined in (1.24), and $F^{(\nu)}$ the associated C-function. Further $\mathcal{U}_{\delta}(\mathcal{N}), \delta \in(0,1)$, denotes the same special neighbourhood of $\Gamma_{\mathcal{N}}$ as given in Lemma 3.1. By [14, Theorem 3.1] and Lemma 3.1 we have

$$
\begin{aligned}
F^{(\nu)}(z) & =\lim _{n \rightarrow \infty} \frac{\Psi_{n-\nu}^{(\nu) *}(z)}{\Phi_{n-\nu}^{(\nu) *}(z)} \\
& =\frac{F(z)\left(\Phi_{\nu}(z)+\Phi_{\nu}^{*}(z)\right)+\left(\Psi_{\nu}(z)-\Psi_{\nu}^{*}(z)\right)}{F(z)\left(\Phi_{\nu}(z)-\Phi_{\nu}^{*}(z)\right)+\left(\Psi_{\nu}(z)+\Psi_{\nu}^{*}(z)\right)}=\frac{\mathcal{G}_{\nu}(z)+z \mathcal{H}_{\nu}(z)}{\mathcal{G}_{\nu}(z)-z \mathcal{H}_{\nu}(z)},
\end{aligned}
$$

where convergence holds uniformly on $\mathcal{U}_{\delta}(\mathcal{N})$ (note that $F^{(\nu)}$ exists on $\Gamma_{\mathcal{N}}$ by $(3.7)$ ). Let us define the functions $\mathcal{G}_{n}^{(\nu)}, \mathcal{H}_{n}^{(\nu)}$ in an analogous way as in (1.29) resp. (1.30), i.e.

$$
\begin{aligned}
& \mathcal{G}_{n}^{(\nu)}(z)=\frac{1}{z^{n}}\left(\Phi_{n}^{(\nu)}(z) F^{(\nu)}(z)+\Psi_{n}^{(\nu)}(z)\right) \\
& \mathcal{H}_{n}^{(\nu)}(z)=\frac{1}{z^{n+1}}\left(\Phi_{n}^{(\nu) *}(z) F^{(\nu)}(z)-\Psi_{n}^{(\nu) *}(z)\right) .
\end{aligned}
$$

Thus there holds for $z \in \mathcal{U}_{\delta}(\mathcal{N})$ by $((1))$

$$
\mathcal{G}_{n-\nu}^{(\nu)}(z)=\frac{1}{z^{n-\nu}}\left(\Phi_{n-\nu}^{(\nu)}(z) \frac{\mathcal{G}_{\nu}(z)+z \mathcal{H}_{\nu}(z)}{\mathcal{G}_{\nu}(z)-z \mathcal{H}_{\nu}(z)}+\Psi_{n-\nu}^{(\nu)}(z)\right) .
$$

Using the fact (cf. [14, (3.7)])

$$
\begin{aligned}
& 2 \Phi_{n}=\sqrt{c_{0}}\left(\left(\Phi_{\nu}+\Phi_{\nu}^{*}\right) \Phi_{n-\nu}^{(\nu)}+\left(\Phi_{\nu}-\Phi_{\nu}^{*}\right) \Psi_{n-\nu}^{(\nu)}\right), \\
& 2 \Psi_{n}=\sqrt{c_{0}}\left(\left(\Psi_{\nu}-\Psi_{\nu}^{*}\right) \Phi_{n-\nu}^{(\nu)}+\left(\Psi_{\nu}+\Psi_{\nu}^{*}\right) \Psi_{n-\nu}^{(\nu)}\right),
\end{aligned}
$$

we obtain (compare the first representation in (1.29))

$$
2 \mathcal{G}_{n}(z)=\frac{\sqrt{c_{0}}}{z^{n-\nu}}\left(\left(\mathcal{G}_{\nu}(z)+z \mathcal{H}_{\nu}(z)\right) \Phi_{n-\nu}^{(\nu)}(z)+\left(\mathcal{G}_{\nu}(z)-z \mathcal{H}_{\nu}(z)\right) \Psi_{n-\nu}^{(\nu)}(z)\right) .
$$

From $((2))$ and $((3))$ there follows

$$
\frac{2}{\sqrt{c_{0}}} \mathcal{G}_{n}(z)=\left(\mathcal{G}_{\nu}(z)-z \mathcal{H}_{\nu}(z)\right) \mathcal{G}_{n-\nu}^{(\nu)}(z)
$$

If we now multiply the identity (2.3) by $\mathcal{G}_{n}$ and use ((4)), (1.25b), (1.35) and the fact that by (1.12) $\alpha_{n}$ and $\lambda_{\nu}^{(n)}$ are uniformly bounded for $n, \nu \in \mathbb{N}_{0}$, we see that 
there exist constants $K_{1}, K_{2} \in \mathbb{R}^{+}$(independent of $n, \nu \in \mathbb{N}_{0}$ and $z=e^{i \varphi}, \varphi \in \mathcal{N}$ ) such that

$((5))$

$$
\begin{aligned}
& \left|\widetilde{\Phi}_{n}(z) \mathcal{G}_{n}(z)\right| \leq K_{1}\left|\Phi_{n}^{*}(z) \mathcal{G}_{n}(z)\right| \\
& \quad+K_{2} \sum_{\nu=0}^{n-1}\left|a_{\nu}-b_{\nu}\right|\left\{\left|\Phi_{n-\nu}^{(\nu) *}(z) \mathcal{G}_{n-\nu}^{(\nu)}(z)\right|+\left|\Psi_{n-\nu}^{(\nu) *}(z) \mathcal{G}_{n-\nu}^{(\nu)}(z)\right|\right\}\left|\widetilde{\Phi}_{\nu}(z) \mathcal{G}_{\nu}(z)\right|
\end{aligned}
$$

for all $n \in \mathbb{N}_{0}$ and $z \in \Gamma_{\mathcal{N}}$.

Next we show that there exists a constant $K_{3} \in \mathbb{R}^{+}$such that for all $n, \nu \in \mathbb{N}_{0}$, $\nu \leq n$, and $z \in \Gamma_{\mathcal{N}}$

$$
\left|\Phi_{n-\nu}^{(\nu) *}(z) \mathcal{G}_{n-\nu}^{(\nu)}(z)\right| \leq K_{3} \quad \text { and } \quad\left|\Psi_{n-\nu}^{(\nu) *}(z) \mathcal{G}_{n-\nu}^{(\nu)}(z)\right| \leq K_{3} .
$$

Then the assertion (3.8) follows from ((5)) and ((6)) by Gronwall's inequality (1.37). To show the estimates $((6))$ we need the following statement (cf. [6, (11.7), p. 17]): If $\mu$ is a distribution function and $F_{\mu}$ denotes the associated C-function for which $\lim _{r \rightarrow 1^{-}} F_{\mu}\left(r e^{i \psi}\right)=F_{\mu}\left(e^{i \psi}\right)$ exists uniformly on $|z|=1$ (or even on a compact subset of $|z|=1$ ), then $\mu$ is uniquely determined by the C-function $F_{\mu}$ by means of the inversion formula $(a \in[0,2 \pi])$

$$
\begin{aligned}
& \frac{\mu(\psi+0)+\mu(\psi-0)}{2}=\mathrm{const}+c_{0}^{\mu} \int_{a}^{\psi} \operatorname{Re} F_{\mu}\left(e^{i \varphi}\right) d \varphi \\
& \text { where } c_{0}^{\mu}=\frac{1}{2 \pi} \int_{a}^{a+2 \pi} d \mu(\varphi) .
\end{aligned}
$$

Since the expressions $e^{-i \frac{\nu}{2} \varphi}\left(\Phi_{\nu}\left(e^{i \varphi}\right)+\Phi_{\nu}^{*}\left(e^{i \varphi}\right)\right), e^{-i \frac{\nu}{2} \varphi}\left(\Psi_{\nu}\left(e^{i \varphi}\right)+\Psi_{\nu}^{*}\left(e^{i \varphi}\right)\right)$, $i e^{-i \frac{\nu}{2} \varphi}\left(\Phi_{\nu}\left(e^{i \varphi}\right)-\Phi_{\nu}^{*}\left(e^{i \varphi}\right)\right)$ and $i e^{-i \frac{\nu}{2} \varphi}\left(\Psi_{\nu}\left(e^{i \varphi}\right)-\Psi_{\nu}^{*}\left(e^{i \varphi}\right)\right)$ are real trigonometric polynomials and since

$$
\operatorname{Re} F\left(e^{i \varphi}\right)=0 \text { for all } \varphi \in \mathcal{N} \text { by (3.3a)) }
$$

(recall $\varphi \notin \operatorname{supp}(\sigma))$, ]we obtain from ((1)) and (3.7)

$$
\operatorname{Re} F^{(\nu)}\left(e^{i \varphi}\right)=0 \text { for all } \nu \in \mathbb{N}_{0}, \varphi \in \mathcal{N} .
$$

Thus by ((7)), note that by $((1)),(3.7)$ and Lemma 3.1(a)

$$
F^{(\nu)}\left(r e^{i \varphi}\right) \underset{r \rightarrow 1^{-}}{\longrightarrow} F^{(\nu)}\left(e^{i \varphi}\right) \text { uniformly on } \Gamma_{\mathcal{N}},
$$

we have for all $\nu \in \mathbb{N}_{0}$

$$
\mathcal{N} \cap \operatorname{supp}\left(\sigma^{(\nu)}\right)=\varnothing .
$$

Moreover $\mathcal{N}$ has a positive distance from $\operatorname{supp}\left(\sigma^{(\nu)}\right)$ (this can be seen from repeating the above argument with $\mathcal{N}$ replaced by $\mathcal{N}_{1}$ which satisfies $\mathcal{N} \subseteq \stackrel{\circ}{\mathcal{N}}_{1} \subset \mathcal{N}_{1} \subset \stackrel{\circ}{\mathcal{M}} \subset$ $\mathcal{M})$. Thus there exists a constant $K_{4} \in \mathbb{R}^{+}$such that for all $\nu \in \mathbb{N}_{0}, \varphi \in \operatorname{supp}\left(\sigma^{(\nu)}\right)$ and $z \in \Gamma_{\mathcal{N}}$

$$
\left|\frac{e^{i \varphi}+z}{e^{i \varphi}-z}\right| \leq K_{4}
$$

From (1.29), (3.3b) (this representation holds also for $\mathcal{G}_{n-\nu}^{(\nu)}$ by $\left.((8))\right),((10))$ and (1.25b) we derive (compare the proof of Theorem 2.2 and note that $\sqrt{c_{0} / c_{0}^{(\nu)}} \Phi_{n-\nu}^{(\nu)}$ 
is orthonormal with respect to $\left.\sigma^{(\nu)}\right)$

$$
\begin{aligned}
& \left|\Phi_{n-\nu}^{(\nu) *}(z) \mathcal{G}_{n-\nu}^{(\nu)}(z)\right| \\
& \leq \frac{1}{2 \pi c_{0}}\{K_{4} \underbrace{\int_{0}^{2 \pi} \frac{c_{0}}{c_{0}^{(\nu)}} \overline{\Phi_{n-\nu}^{(\nu)}\left(e^{i \varphi}\right)} \Phi_{n-\nu}^{(\nu)}\left(e^{i \varphi}\right) d \sigma^{(\nu)}(\varphi)}_{=2 \pi} \\
& \left.+|\underbrace{\frac{c_{0}}{c_{0}^{\left(\nu_{0}\right)}} \int_{0}^{2 \pi} \frac{\frac{1}{e^{i \varphi}}+\frac{1}{z}}{\frac{1}{e^{i \varphi}}-\frac{1}{z}}\left(\bar{\Phi}_{n-\nu}^{(\nu)}\left(\frac{1}{e^{i \varphi}}\right)-\bar{\Phi}_{n-\nu}^{(\nu)}\left(\frac{1}{z}\right)\right) \Phi_{n-\nu}^{(\nu)}\left(e^{i \varphi}\right) d \sigma^{(\nu)}(\varphi)}_{=2 \pi}|\right\} .
\end{aligned}
$$

Thus we have shown the first part of the assertion $((6))$.

Now let $\sigma_{\Psi}^{(\nu)}$ denote the distribution function with respect to $\left\{\Psi_{n}^{(\nu)}\right\}_{n \in \mathbb{N}_{0}}$ (note that $\left\{\Psi_{n}^{(\nu)}\right\}_{n \in \mathbb{N}_{0}}$ is generated by the recurrence coefficients $\left\{-a_{n+\nu}\right\}_{n \in \mathbb{N}_{0}}$; thus $\sigma_{\Psi}^{(\nu)}$ exists by $\left[6\right.$, Theorem 13.3, p. 21]) and $F_{\Psi}^{(\nu)}$ the associated C-function. By

$$
F_{\Psi}^{(\nu)}(z)=\lim _{n \rightarrow \infty} \frac{\Phi_{n-\nu}^{(\nu) *}(z)}{\Psi_{n-\nu}^{(\nu) *}(z)}=\frac{\mathcal{G}_{\nu}(z)-z \mathcal{H}_{\nu}(z)}{\mathcal{G}_{\nu}(z)+z \mathcal{H}_{\nu}(z)}=\frac{1}{F^{(\nu)}(z)}
$$

uniformly on $\mathcal{U}_{\delta}(\mathcal{N})$ (compare [13, (1.13), p. 158], ((1)) and note (3.7)) we get

$$
\mathcal{G}_{\Psi, n-\nu}^{(\nu)}(z):=\frac{1}{z^{n-\nu}}\left(\Psi_{n-\nu}^{(\nu)}(z) F_{\Psi}^{(\nu)}(z)+\Phi_{n-\nu}^{(\nu)}(z)\right)=\frac{1}{F^{(\nu)}(z)} \mathcal{G}_{n-\nu}^{(\nu)}(z)
$$

and therefore

$$
\left|\Psi_{n-\nu}^{(\nu) *}(z) \mathcal{G}_{n-\nu}^{(\nu)}(z)\right|=\left|F^{(\nu)}(z)\right| \cdot\left|\Psi_{n-\nu}^{(\nu) *}(z) \mathcal{G}_{\Psi, n-\nu}^{(\nu)}(z)\right| .
$$

By $((8))$ and $((11))$ we have $\operatorname{Re} F_{\Psi}^{(\nu)}\left(e^{i \varphi}\right)=0, \varphi \in \mathcal{N}$, and thus

$$
\mathcal{N} \cap \operatorname{supp}\left(\sigma_{\Psi}^{(\nu)}\right)=\varnothing \quad(\text { compare }((9)))
$$

(more exactly, $\mathcal{N}$ has again a positive distance from $\operatorname{supp}\left(\sigma_{\Psi}^{(\nu)}\right)$; compare the lines after $((9)))$. Now we can show the uniform boundedness of $\Psi_{n-\nu}^{(\nu) *} \mathcal{G}_{n-\nu}^{(\nu)}$ on $\Gamma_{\mathcal{N}}$ in the same way as we have shown the boundedness of $\Phi_{n-\nu}^{(\nu) *} \mathcal{G}_{n-\nu}^{(\nu)}$ by using $((12))$ and (3.7).

(b) Now $\mathcal{N} \subseteq \operatorname{supp}(\sigma)$. Since the polynomials $\Phi_{n}, \Psi_{n}$ are uniformly bounded on $\Gamma_{\mathcal{N}}$, we have the uniform boundedness of $\widetilde{\Phi}_{n}(z)$ on $\Gamma_{\mathcal{N}}$ by Proposition 3.1 and by assumption (3.1) the function $F$ exists on $\Gamma_{\mathcal{N}}$ and is uniformly bounded there. Thus we have the uniform boundedness of

$$
\widetilde{\Phi}_{n}(z) \mathcal{G}_{n}(z)=\frac{\widetilde{\Phi}_{n}(z)}{z^{n}}\left(\Phi_{n}(z) F(z)+\Psi_{n}(z)\right)
$$

on $\Gamma_{\mathcal{N}}$.

Remark 3.1. Concerning the assumptions of Lemma 3.2, which will be assumed in the following theorems, let us note that

(a) From the proof of Lemma 3.2(a) and by assumption (3.7) we see that $\operatorname{Re} \frac{\mathcal{G}_{n}(z)+z \mathcal{H}_{n}(z)}{\mathcal{G}_{n}(z)-z \mathcal{H}_{n}(z)}=0$ for $z \in \Gamma_{\mathcal{N}}($ recall $\mathcal{N} \cap \operatorname{supp}(\sigma)=\varnothing)$; thus

$$
\left|\frac{\mathcal{G}_{n}(z)+z \mathcal{H}_{n}(z)}{\mathcal{G}_{n}(z)-z \mathcal{H}_{n}(z)}\right|=\left|\operatorname{Im} \frac{\mathcal{G}_{n}(z)+z \mathcal{H}_{n}(z)}{\mathcal{G}_{n}(z)-z \mathcal{H}_{n}(z)}\right| .
$$


(b) Assumption (3.7) is satisfied, if and only if the set $\Gamma_{\mathcal{M}}$, where $\mathcal{M}$ is given as in Lemma 3.2(a), contains no zeros of $\mathcal{G}_{n} \pm z \mathcal{H}_{n}$ (i.e. $\Gamma_{\mathcal{N}}$ has a positive distance from these zeros): First note that $\mathcal{G}_{n}+z \mathcal{H}_{n}$ and $\mathcal{G}_{n}-z \mathcal{H}_{n}$ have no common zero on $\Gamma_{\mathcal{M}}$. Indeed, assume the opposite: $\mathcal{G}_{n}(z)+z \mathcal{H}_{n}(z)=\mathcal{G}_{n}(z)-z \mathcal{H}_{n}(z)=0$ for a fixed $n \in \mathbb{N}_{0}$ and $z \in \Gamma_{\mathcal{M}}$; i.e. $\mathcal{G}_{n}(z)=0$ and $\mathcal{H}_{n}(z)=0$ (by (1.35)) and thus by (1.29) and (1.30) $\Phi_{n}^{*}(z) \Psi_{n}(z)+\Phi_{n}(z) \Psi_{n}^{*}(z)=0$, which contradicts (1.28) (note $|z|=1)$. Further the points $((1)),((9)),((10))$ and $((13))$ in the proof of Lemma 3.2 remain valid, if there are no zeros of $\mathcal{G}_{n} \pm z \mathcal{H}_{n}$ in $\Gamma_{\mathcal{M}}$. Thus we have

$$
\begin{aligned}
& \left|F^{(n)}(z)\right|=\left|\frac{1}{2 \pi c_{0}^{(n)}} \int_{0}^{2 \pi} \frac{e^{i \varphi}+z}{e^{i \varphi}-z} d \sigma^{(n)}(\varphi)\right| \leq K_{4} \\
& \left|F_{\Psi}^{(n)}(z)\right|=\left|\frac{1}{2 \pi c_{\Psi, 0}^{(n)}} \int_{0}^{2 \pi} \frac{e^{i \varphi}+z}{e^{i \varphi}-z} d \sigma_{\Psi}^{(n)}(\varphi)\right| \leq K_{4} \quad \text { for all } n \in \mathbb{N}_{0}, z \in \Gamma_{\mathcal{N}}
\end{aligned}
$$

and assumption (3.7) follows from ((1)).

From $((1))$ and $((7))$ in the proof of Lemma 3.2(a) it can be seen that the zeros of $\mathcal{G}_{n}-z \mathcal{H}_{n}$ (resp. $\mathcal{G}_{n}+z \mathcal{H}_{n}$ ) correspond to singularity-points resp. mass-points of $\sigma^{(n)}$ (resp. $\sigma_{\Psi}^{(n)}$ - given as in the proof of Lemma 3.2).

(c) Concerning the uniform boundedness of $\Phi_{n}, \Psi_{n}$ on compact subsets of $\operatorname{supp}(\sigma)$, assumed in Lemma 3.2(b), let us mention the following facts:

(c1) By [21, Theorem 4, p. 151] we know that $\Phi_{n}$ is bounded on a set $\Gamma_{\mathcal{N}}$ if $\sigma$ is absolutely continuous on $[0,2 \pi]$ with $\sigma^{\prime}>0$ a.e. on $[0,2 \pi]$, where $\sigma^{\prime}$ is of Dini-Lipschitz type on $\mathcal{N}$ and $\inf _{\varphi \in \mathcal{N}} \sigma^{\prime}(\varphi)>0$.

(c2) It is very likely that uniform boundedness of orthogonal polynomials on compact subsets of $\operatorname{supp}(\sigma)$ is given, if the weight function is positive on these sets and "behaves nicely" there. For instance we have shown in [15] that polynomials $\left\{\Phi_{n}\right\}_{n \in \mathbb{N}_{0}}$ orthonormal with respect to an absolute continuous measure $\sigma$, the support of which consists of a finite number of arcs, are bounded on such compact subsets of $\operatorname{Int}(\operatorname{supp}(\sigma))$, on which $\sigma^{\prime}$ fulfills a Lipschitz condition.

Theorem 3.1. Suppose that the assumptions of Lemma 3.2(a) or Lemma 3.2(b) are fulfilled. Then we have

$$
\lim _{n \rightarrow \infty}\left(\widetilde{\Phi}_{n}^{*}(z) \mathcal{G}_{n}(z)-z \widetilde{\Phi}_{n}(z) \mathcal{H}_{n}(z)\right)=: \Delta(z)
$$

exists uniformly on $\Gamma_{\mathcal{N}}$.

Proof. If $\mathcal{N}$ is a subset of $\operatorname{supp}(\sigma)$, we can see as in the proof of Lemma 3.2(b) by using (1.25b) that under the assumptions of Lemma 3.2(b) the sequences $\left\{\widetilde{\Phi}_{n} \mathcal{H}_{n}\right\}_{n \in \mathbb{N}_{0}}$, $\left\{\widetilde{\Phi}_{n}^{*} \mathcal{H}_{n}\right\}_{n \in \mathbb{N}_{0}}$ and $\left\{\widetilde{\Phi}_{n} \mathcal{G}_{n}\right\}_{n \in \mathbb{N}_{0}}$ are uniformly bounded on $\Gamma_{\mathcal{N}}$. If $|z|=1$ and $z \notin\left\{e^{i \varphi}: \varphi \in \operatorname{supp}(\sigma)\right\}$ we have $\left|\mathcal{G}_{n}(z)\right|=\left|\mathcal{H}_{n}(z)\right|$ by (1.35) for all $n \in \mathbb{N}_{0}$, and we see the uniform boundedness of the above sequences by Lemma 3.2(a), (1.25b), (1.35) and (1.12)

Now (3.9) can be derived from (2.2) and (1.12) as in the proof of (2.7) in Theorem 2.2 .

Now the question arises quite naturally, under which conditions on $\sigma$ resp. on the system of orthogonal polynomials $\left\{\Phi_{n}\right\}_{n \in \mathbb{N}_{0}}$ do the limit relations (2.5) and (2.8) also hold for $|z|=1$. If $a_{n} \rightarrow 0$ (and some further assumptions on $\tilde{\sigma}$ mentioned in Section 1 ) this question, concerning (2.8), is already answered in a positive sense by Maté, Nevai, Totik [9, Theorem 3, p. 76] and Rakhmanov [21, Theorem 2, p. 156] 
(compare (1.18)). Since by (1.19) $a_{n} \rightarrow 0$ implies that $\operatorname{supp}(\sigma)=[0,2 \pi]$, the case that $\operatorname{supp}(\sigma)$ is a subset of $(0,2 \pi)$ remains, in particular, to be considered. Hence let us assume that there exists a compact set $\mathcal{N} \subseteq[0,2 \pi]$, which has a positive distance from $\operatorname{supp}(\sigma)$. It can be shown by using the same methods introduced by Golinskii, Nevai and Van Assche in $[12, \S 3]$ that the accumulation points of $\operatorname{supp}(\sigma)$ and $\operatorname{supp}(\tilde{\sigma})$ are indentical. ${ }^{1}$ Now we can assume that the set $\mathcal{N}$ has no common point with $\operatorname{supp}(\sigma)$ and $\operatorname{supp}(\tilde{\sigma})$. Thus we can define the functions of the second kind $\widetilde{\mathcal{G}}_{n}$ and $\widetilde{\mathcal{H}}_{n}, n \in \mathbb{N}_{0}$, with respect to $\tilde{\sigma}$ in the same way as in (1.29), (1.30), resp. (3.3b), (3.3c). The following theorem gives conditions under which the asymptotic relations (2.5) and (2.8) hold uniformly for $z \in \Gamma_{\mathcal{N}}$, if $\mathcal{N} \cap \operatorname{supp}(\sigma)=\varnothing$.

Theorem 3.2. Suppose that (1.12) holds and let $\mathcal{N} \subset \stackrel{\circ}{\mathcal{M}}$, where $\mathcal{N}$ and $\mathcal{M}$ are compact subsets of $[0,2 \pi], \mathcal{M} \cap \operatorname{supp}(\sigma)=\varnothing, \mathcal{M} \cap \operatorname{supp}(\tilde{\sigma})=\varnothing$ and $\Gamma_{\mathcal{M}}$ contains no zeros of $\mathcal{G}_{n} \pm z \mathcal{H}_{n}, \widetilde{\mathcal{G}}_{n} \pm z \widetilde{\mathcal{H}}_{n}, n \in \mathbb{N}_{0}$ (compare the above considerations in Remark 3.1(a)). Further assume that

$$
\frac{1}{\left|\Phi_{n+1}^{*}(z)\right|^{2}} \sum_{\nu=0}^{n}\left|\Phi_{\nu}^{*}(z)\right|^{2} \text { is uniformly bounded on } \Gamma_{\mathcal{N}} \text {. }
$$

Then we have uniformly on $\Gamma_{\mathcal{N}}$

$$
\lim _{n \rightarrow \infty}\left(\frac{\Phi_{n}(z)}{\Phi_{n}^{*}(z)}-\frac{\widetilde{\Phi}_{n}(z)}{\widetilde{\Phi}_{n}^{*}(z)}\right)=0
$$

and

$$
\lim _{n \rightarrow \infty}\left(\frac{\widetilde{\Phi}_{n}^{*}(z)}{\Phi_{n}^{*}(z)}-\frac{c_{0}}{2} \Delta(z)\right)=0,
$$

where $\Delta(z)$ is defined as in Theorem 3.1 .

Proof. Since $\Phi_{n}^{*}(z) \neq 0$ for $z \in \Gamma_{\mathcal{N}}$, we can write

$$
\left|\widetilde{\Phi}_{n}^{*}(z) \mathcal{G}_{n}(z)\right|=\left|\frac{\widetilde{\Phi}_{n}^{*}(z)}{\Phi_{n}^{*}(z)}\right|\left|\Phi_{n}^{*}(z) \mathcal{G}_{n}(z)\right| .
$$

By Lemma 3.2(a), (1.25b) and (1.12) $\left\{\widetilde{\Phi}_{n}^{*} \mathcal{G}_{n}\right\}_{n \in \mathbb{N}_{0}}$ is uniformly bounded on $\Gamma_{\mathcal{N}}$. Further there holds

$$
\left|\Phi_{n}^{*}(z) \mathcal{G}_{n}(z)\right| \geq \frac{1}{c_{0}} \quad \text { for all } n \in \mathbb{N}_{0}, z \in \Gamma_{\mathcal{N}}
$$

Indeed, assume the opposite, i.e. $\left|\Phi_{n}^{*}(z) \mathcal{G}_{n}(z)\right|<1 / c_{0}$ for a fixed $n \in \mathbb{N}_{0}$ and $z \in \Gamma_{\mathcal{N}}$. Then we have by $(1.25 \mathrm{~b})$ and $(1.35)$

$$
\left|\Phi_{n}^{*}(z) \mathcal{G}_{n}(z)-z \Phi_{n}(z) \mathcal{H}_{n}(z)\right| \leq 2\left|\Phi_{n}^{*}(z) \mathcal{G}_{n}(z)\right|<\frac{2}{c_{0}}
$$

which contradicts (1.36). Thus we obtain from $((1))$ that $\left\{\widetilde{\Phi}_{n}^{*} / \Phi_{n}^{*}\right\}_{n \in \mathbb{N}_{0}}$ is uniformly bounded on $\Gamma_{\mathcal{N}}$; i.e. there exists a constant $K \in \mathbb{R}^{+}$such that

$$
\left|\frac{\widetilde{\Phi}_{n}^{*}(z)}{\Phi_{n}^{*}(z)}\right| \leq K<\infty \quad \text { for all } n \in \mathbb{N}_{0}, z \in \Gamma_{\mathcal{N}} .
$$

\footnotetext{
${ }^{1}$ This fact, which makes the statement and the proof of the following theorem much more elegant than the original one, was not known to us before Golinskii, Nevai and Van Assche sent us their manuscript [12].
} 
Now we can change the roles of $\left\{a_{n}\right\}_{n \in \mathbb{N}_{0}}$ and $\left\{b_{n}\right\}_{n \in \mathbb{N}_{0}}$ (note that, by the definition of the set $\mathcal{M}$ and Remark 3.1(b), the assertions of Lemma 3.2(a) hold for $\sigma$ and $\tilde{\sigma}$ resp. $\left\{\Phi_{n}\right\}_{n \in \mathbb{N}_{0}}$ and $\left.\left\{\widetilde{\Phi}_{n}\right\}_{n \in \mathbb{N}_{0}}\right)$, and we obtain as in $((2))$

$$
0<k \leq\left|\frac{\widetilde{\Phi}_{n}^{*}(z)}{\Phi_{n}^{*}(z)}\right| \leq K<\infty \quad \text { for all } n \in \mathbb{N}_{0}, z \in \Gamma_{\mathcal{N}},
$$

i.e.

$$
k\left|\Phi_{n}^{*}(z)\right| \leq\left|\widetilde{\Phi}_{n}^{*}(z)\right| \leq K\left|\Phi_{n}^{*}(z)\right| .
$$

Now by $((4))$ we obtain on $\Gamma_{\mathcal{N}}$

$$
\frac{1}{\left|\widetilde{\Phi}_{n+1}^{*}(z)\right|^{2}} \sum_{\nu=0}^{n}\left|\widetilde{\Phi}_{\nu}^{*}(z)\right|^{2} \leq \frac{K^{2}}{k^{2}\left|\Phi_{n+1}^{*}(z)\right|^{2}} \sum_{\nu=0}^{n}\left|\Phi_{\nu}^{*}(z)\right|^{2} .
$$

Thus by (3.10) there exists a constant $K_{1} \in \mathbb{R}^{+}$such that uniformly on $\Gamma_{\mathcal{N}}$

$$
\frac{1}{\left|\widetilde{\Phi}_{n+1}^{*}(z)\right|^{2}} \sum_{\nu=0}^{n}\left|\widetilde{\Phi}_{\nu}^{*}(z)\right|^{2} \leq K_{1} \quad \text { for all } n \in \mathbb{N}_{0}
$$

By (3.10) and ((5)), compare also the proof of Theorem 2.1, we have $\left(\varepsilon_{\nu}=\left|a_{\nu}-b_{\nu}\right|\right)$

$$
\frac{1}{\left|\Phi_{n+1}^{*}(z)\right|^{2}} \sum_{\nu=0}^{n} \varepsilon_{\nu}\left|\Phi_{\nu}^{*}(z)\right|^{2} \cdot \frac{1}{\left|\tilde{\Phi}_{n+1}^{*}(z)\right|^{2}} \sum_{\nu=0}^{n} \varepsilon_{\nu}\left|\tilde{\Phi}_{\nu}^{*}(z)\right|^{2} \underset{n \rightarrow \infty}{\rightarrow} 0
$$

uniformly on $\Gamma_{\mathcal{N}}$. Now (3.11) follows from the estimate $((2))$ in the proof of Theorem 2.1. With the abbreviation $\Delta_{n}(z):=\widetilde{\Phi}_{n}^{*}(z) \mathcal{G}_{n}(z)-z \widetilde{\Phi}_{n}(z) \mathcal{H}_{n}(z)$ we get by $(1.36)$

$$
\Delta_{n}(z)=\frac{\widetilde{\Phi}_{n}^{*}(z)}{\Phi_{n}^{*}(z)}\left(\frac{2}{c_{0}}+z \Phi_{n}^{*}(z) \mathcal{H}_{n}(z)\left(\frac{\Phi_{n}(z)}{\Phi_{n}^{*}(z)}-\frac{\widetilde{\Phi}_{n}(z)}{\widetilde{\Phi}_{n}^{*}(z)}\right)\right) .
$$

Now the assertion (3.12) follows immediately from $((3))$, the uniform boundedness of $\left\{\Phi_{n}^{*} \mathcal{H}_{n}\right\}_{n \in \mathbb{N}_{0}}$ (compare $((6))$ in the proof of Lemma 3.2 with $\nu=0$ and note (1.25b) resp. (1.35)), from (3.11) and Theorem 3.1

Remark 3.2. (a) It is very likely that assumption (3.10) holds under very weak conditions on $\sigma$, as in the asymptotically periodic case (cf. [17]).

(b) Let us point out that the limit relations (3.11) and (3.12) will not hold in general on subsets of $\operatorname{supp}(\sigma)$ as the asymptotic periodic case [17] or the case of finite perturbations (i.e. $a_{n}=b_{n}$ for $n \geq n_{0}$ ) [14] shows. Thus from (3.12) the fundamental difference to the case $a_{n} \rightarrow 0$ can be seen, where (under further suitable assumptions on $\sigma$ and $\tilde{\sigma}$ given in Section 1 ) the analog limit relation (1.18) holds on subsets of $\operatorname{supp}(\sigma)($ compare also $(2.9))$.

We now show how the orthogonality measures $d \sigma$ and $d \tilde{\sigma}$ are related to each other, if (1.12) is fulfilled. Recall that the $\widetilde{\Phi}_{n}$ 's, $n \in \mathbb{N}_{0}$, are not orthonormal with respect to $d \tilde{\sigma}$ in general, because we have normed $\widetilde{\Phi}_{n}$ such that $\widetilde{\Phi}_{n}$ and $\Phi_{n}$ have the same leading coefficient. We denote by $\widetilde{\Phi}_{n}^{\text {ON }}$ the orthonormal polynomials with respect to $d \tilde{\sigma}$. From $(1.2),(1.4),(1.9)$ and (1.12) we obtain for the factor in the 
representation (1.11)

$$
\lim _{n \rightarrow \infty}\left(\frac{c_{0}}{\tilde{c}_{0}} \prod_{j=0}^{n-1} \frac{1-\left|a_{j}\right|^{2}}{1-\left|b_{j}\right|^{2}}\right)=: \beta^{2} \in \mathbb{R}^{+}
$$

For the following theorem compare Theorem 3 in [11].

Theorem 3.3. Let $\mathcal{N}$ be a compact subset of $\operatorname{supp}(\sigma)$ and suppose that the assumptions of Lemma 3.2(b) are fulfilled. Then there holds

$$
\left|\frac{\beta c_{0}}{2} \Delta\left(e^{i \varphi}\right)\right|^{2}=\frac{d \sigma(\varphi)}{d \tilde{\sigma}(\varphi)} \quad \text { for almost every } \varphi \in \mathcal{N}
$$

where $\Delta$ and $\beta$ are defined as in (3.9) and (3.13).

Proof. From (1.1) and (1.5) we have

$$
z \Phi_{n}(z)=\sqrt{1-\left|a_{n}\right|^{2}} \Phi_{n+1}(z)+\bar{a}_{n} \Phi_{n}^{*}(z) .
$$

Let $m \in \mathbb{Z}$ be fixed. Using the above recurrence relation and the orthogonality properties of $\Phi_{n}, n \in \mathbb{N}_{0}$, one obtains (after some straightforward calculation)

$$
\int_{0}^{2 \pi} e^{i m \varphi} \Phi_{n}\left(e^{i \varphi}\right) \overline{\Phi_{n}\left(e^{i \varphi}\right)} d \sigma(\varphi)=q_{m}\left(a_{n+|m|-1}, \ldots, a_{n-|m|}\right),
$$

where $q_{m}$ is a continuous function only depending on the $2 m$ recurrence coefficients $a_{n-|m|}, \ldots, a_{n+|m|-1}$. Just as well we have

$$
\int_{0}^{2 \pi} e^{i m \varphi} \widetilde{\Phi}_{n}^{\mathrm{ON}}\left(e^{i \varphi}\right) \overline{\widetilde{\Phi}_{n}^{\mathrm{ON}}\left(e^{i \varphi}\right)} d \tilde{\sigma}(\varphi)=q_{m}\left(b_{n+|m|-1}, \ldots, b_{n-|m|}\right) .
$$

In view of (1.12) this means

$$
\begin{aligned}
\lim _{n \rightarrow \infty}\left(\int_{0}^{2 \pi} Q\left(e^{i \varphi}\right) \Phi_{n}\left(e^{i \varphi}\right) \overline{\Phi_{n}\left(e^{i \varphi}\right)}\right. & d \sigma(\varphi) \\
& \left.-\int_{0}^{2 \pi} Q\left(e^{i \varphi}\right) \widetilde{\Phi}_{n}^{\mathrm{ON}}\left(e^{i \varphi}\right) \overline{\widetilde{\Phi}_{n}^{\mathrm{ON}}\left(e^{i \varphi}\right)} d \tilde{\sigma}(\varphi)\right)=0
\end{aligned}
$$

for every Laurent-polynomial $Q$. Thus there follows from the Banach-Steinhaus Theorem

$((1))$

$$
\lim _{n \rightarrow \infty}\left(\int_{\mathcal{N}} g(\varphi) \Phi_{n}\left(e^{i \varphi}\right) \overline{\Phi_{n}\left(e^{i \varphi}\right)} d \sigma(\varphi)-\int_{\mathcal{N}} g(\varphi) \widetilde{\Phi}_{n}^{\mathrm{ON}}\left(e^{i \varphi}\right) \overline{\widetilde{\Phi}_{n}^{\mathrm{ON}}\left(e^{i \varphi}\right)} d \tilde{\sigma}(\varphi)\right)=0
$$

for every $2 \pi$-periodic, continuous function $g$ that vanishes at the end points of $\mathcal{N}$. Note that the integrals in $((1))$ exist for all such functions $g$ because the sequence $\left\{\Phi_{n}\right\}_{n \in \mathbb{N}_{0}}$ is uniformly bounded on $\Gamma_{\mathcal{N}}$ by assumption and $\left\{\widetilde{\Phi}_{n}^{\text {ON }}\right\}_{n \in \mathbb{N}_{0}}$ is uniformly bounded by Proposition 3.1 and (3.13). Further we know that the sequences $\left\{\Psi_{n}\right\}_{n \in \mathbb{N}_{0}}$ and $\left\{\widetilde{\Psi}_{n}^{\mathrm{ON}}\right\}_{n \in \mathbb{N}_{0}}$ are uniformly bounded on $\Gamma_{\mathcal{N}}$ as well. Thus by the fact that $c_{0}\left|\Phi_{n}\left(e^{i \varphi}\right)\right| \geq 1 /\left|\Psi_{n}\left(e^{i \varphi}\right)\right|, \tilde{c}_{0}\left|\widetilde{\Phi}_{n}^{\mathrm{ON}}\left(e^{i \varphi}\right)\right| \geq 1 /\left|\widetilde{\Psi}_{n}^{\mathrm{ON}}\left(e^{i \varphi}\right)\right|$, which follows from (1.28), one can derive from [10, formulae (7) and (11)] that there cannot appear point masses at points from $\mathcal{N}$. Hence $((1))$ holds also true for every continuous function $g$ on $\mathcal{N}$, which can easily be seen by approximating $g$ by a 
continuous function which vanishes at the end points of $\mathcal{N}$. In an analogous way we see that there also hold

$((2))$

$$
\begin{aligned}
& \lim _{n \rightarrow \infty}\left(\int_{\mathcal{N}} g(\varphi) \Phi_{n}^{*}\left(e^{i \varphi}\right) \overline{\Phi_{n}\left(e^{i \varphi}\right)} d \sigma(\varphi)-\int_{\mathcal{N}} g(\varphi) \widetilde{\Phi}_{n}^{\mathrm{ON} *}\left(e^{i \varphi}\right) \overline{\widetilde{\Phi}_{n}^{\mathrm{ON}}\left(e^{i \varphi}\right)} d \tilde{\sigma}(\varphi)\right)=0, \\
& \lim _{n \rightarrow \infty}\left(\int_{\mathcal{N}} g(\varphi) \overline{\Phi_{n}^{*}\left(e^{i \varphi}\right)} \Phi_{n}\left(e^{i \varphi}\right) d \sigma(\varphi)-\int_{\mathcal{N}} g(\varphi) \overline{\widetilde{\Phi}_{n}^{\mathrm{ON} *}\left(e^{i \varphi}\right)} \widetilde{\Phi}_{n}^{\mathrm{ON}}\left(e^{i \varphi}\right) d \tilde{\sigma}(\varphi)\right)=0 .
\end{aligned}
$$

Next let us mention that the functions $\mathcal{G}_{n}$ and $\mathcal{H}_{n}$ are uniformly bounded on $\Gamma_{\mathcal{N}}$ (compare the proof of Lemma 3.2(b) and (1.25b)). Thus from $((1)),((2))$ and again from the Banach-Steinhaus Theorem there follows that the difference of

$$
\int_{\mathcal{N}} g(\varphi)\left|\widetilde{\Phi}_{n}^{\mathrm{ON} *}\left(e^{i \varphi}\right) \mathcal{G}_{n}\left(e^{i \varphi}\right)-z \widetilde{\Phi}_{n}^{\mathrm{ON}}\left(e^{i \varphi}\right) \mathcal{H}_{n}\left(e^{i \varphi}\right)\right|^{2} d \tilde{\sigma}(\varphi)
$$

and

$$
\int_{\mathcal{N}} g(\varphi)|\underbrace{\Phi_{n}^{*}\left(e^{i \varphi}\right) \mathcal{G}_{n}\left(e^{i \varphi}\right)-z \Phi_{n}\left(e^{i \varphi}\right) \mathcal{H}_{n}\left(e^{i \varphi}\right)}_{\left.=\frac{2}{c_{0}} \text { (by }(1.36)\right)}|^{2} d \sigma(\varphi)
$$

converges to zero as $n \rightarrow \infty$. Since

$$
\widetilde{\Phi}_{n}^{\mathrm{ON} *}\left(e^{i \varphi}\right) \mathcal{G}_{n}\left(e^{i \varphi}\right)-z \widetilde{\Phi}_{n}^{\mathrm{ON}}\left(e^{i \varphi}\right) \mathcal{H}_{n}\left(e^{i \varphi}\right) \underset{n \rightarrow \infty}{\longrightarrow} \beta \cdot \Delta\left(e^{i \varphi}\right)
$$

uniformly on $\Gamma_{\mathcal{N}}$ by Theorem 3.1 there follows from $((3))$ and $((4))$

$$
\beta^{2} \int_{\mathcal{N}} g(\varphi)\left|\Delta\left(e^{i \varphi}\right)\right|^{2} d \tilde{\sigma}(\varphi)=\frac{4}{c_{0}^{2}} \int_{\mathcal{N}} g(\varphi) d \sigma(\varphi)
$$

This proves the theorem.

Remark 3.3. If $\left\{\Phi_{n}\right\}_{n \in \mathbb{N}_{0}}$ and $\left\{\widetilde{\Phi}_{n}\right\}_{n \in \mathbb{N}_{0}}$ belong to the Szegö-class, then, in view of (2.9) and (3.9), Theorem 3.3 corresponds to the relationship

$$
\left|D\left(e^{i \varphi}, \frac{\sigma^{\prime}}{\widetilde{\sigma}^{\prime}}\right)\right|^{2}=\frac{\sigma^{\prime}(\varphi)}{\tilde{\sigma}^{\prime}(\varphi)} \quad \text { a.e. on }[0,2 \pi] .
$$

Note that (3.15) follows immediately from (1.16) and the fact that $D\left(z, \sigma^{\prime} / \tilde{\sigma}^{\prime}\right)=$ $D(z, \sigma) / D\left(z, \tilde{\sigma}^{\prime}\right)$.

If the recurrence coefficients of $\left\{\Phi_{n}\right\}_{n \in \mathbb{N}_{0}}$ satisfy the weaker condition $a_{n} \rightarrow 0$ and $\sigma$ and $\tilde{\sigma}$ fulfill the assumptions assumed in (1.18) and in the lines after (1.18), then (3.14) can also be derived from (1.18) (which holds now on $|z|=1$ ) and $[8$, Theorem 5, p. 60]

Since there are no asymptotics at all if $\lim _{n \rightarrow \infty} a_{n}$ does not exist, it's of interest whether there holds a relation of the type (1.18). Let us point out, see (1.15), that there is no "classical" Szegö-function if $\operatorname{supp}(\sigma)$ consists for instance of several disjoint arcs. Thus the question arises how the function $\Delta$ can be expressed in terms of $\sigma$ and $\tilde{\sigma}$. With the help of Theorem 2.2 and Theorems 3.1-3.3 we are able to settle this question.

Therefore let $0=: \varphi_{0}<\varphi_{1}<\cdots<\varphi_{2 l} \leq \varphi_{2 l+1}:=2 \pi, l \in \mathbb{N}$, and

$$
E_{l}:=\bigcup_{j=1}^{l}\left[\varphi_{2 j-1}, \varphi_{2 j}\right], \quad \Gamma_{l}:=\left\{e^{i \varphi}: \varphi \in E_{l}\right\} .
$$


Further let $R \in \mathbb{P}_{2 l}^{\mathbb{C}}$ be that (up to a positive multiplicative constant) uniquely determined complex polynomial, which satisfies (compare $[15,17]$ )

$$
\begin{gathered}
R=R^{*}, \\
R\left(e^{i \varphi_{j}}\right)=0, \quad j=1, \ldots, 2 l, \\
e^{-i l \varphi} R\left(e^{i \varphi}\right)<0, \quad \varphi \in\left(\varphi_{2 j-1}, \varphi_{2 j}\right), j=1, \ldots, l
\end{gathered}
$$

(note that $e^{-i l \varphi} R\left(e^{i \varphi}\right)$ is a real trigonometric polynomial). In what follows we choose that branch of $\sqrt{R}$ that is analytic on $\mathbb{C} \backslash \Gamma_{l}$ and is such that

$$
\operatorname{sgn} \sqrt{R\left(e^{i \varphi}\right)}=(-1)^{j} e^{i \frac{l}{2} \varphi}, \quad \varphi \in\left(\varphi_{2 j}, \varphi_{2 j+1}\right), j=0, \ldots, l .
$$

Finally let us define the real function $r(\varphi)$ on $E_{l}$ by

$$
r(\varphi):=(-1)^{j} \sqrt{\left|R\left(e^{i \varphi}\right)\right|}, \quad \varphi \in\left[\varphi_{2 j-1}, \varphi_{2 j}\right], j=1, \ldots, l .
$$

The following theorem holds.

Theorem 3.4. Let $E_{l}, R$ and $r$ be defined as in (3.16)-(3.19) and let $\mathcal{B}$ be the closed unit disk. Suppose that $\operatorname{supp}(\sigma)=\operatorname{supp}(\tilde{\sigma})=E_{l}$ and that $\sigma, \tilde{\sigma}$ are absolutely continuous on $E_{l}$ and satisfy $0<m \leq \sigma^{\prime}(\varphi) / \tilde{\sigma}^{\prime}(\varphi) \leq M<\infty$ on $E_{l}$. Furthermore assume that the assumptions of the Theorems 2.2 and 3.2 are fulfilled on each compact subset of $\mathcal{B} \backslash \Gamma_{l}$ and that (3.9) holds uniformly on $E_{l}$. Then the function $\Delta$ defined in (2.7) and (3.9) has the following representation

$$
\begin{gathered}
\Delta(z)=\gamma^{-1} \exp \left\{\frac{1}{4 \pi i} \sqrt{z^{-l} R(z)} \int_{E_{l}} \frac{e^{i \varphi}+z}{e^{i \varphi}-z} \ln \frac{\sigma^{\prime}(\varphi)}{\tilde{\sigma}^{\prime}(\varphi)} \frac{d \varphi}{r(\varphi)}\right\} \\
\text { for } z \in \mathcal{B} \backslash \Gamma_{l} \text { and l even, }
\end{gathered}
$$

respectively

$$
\begin{gathered}
\Delta(z)=\gamma^{-1} \exp \left\{\frac{1}{2 \pi i} \sqrt{z^{-l+1} R(z)} \int_{E_{l}} \frac{e^{i \frac{\varphi}{2}}}{e^{i \varphi}-z} \ln \frac{\sigma^{\prime}(\varphi)}{\tilde{\sigma}^{\prime}(\varphi)} \frac{d \varphi}{r(\varphi)}\right\} \\
\text { for } z \in \mathcal{B} \backslash \Gamma_{l} \text { and } l \text { odd },
\end{gathered}
$$

where $\gamma=\beta c_{0} / 2$ and $\beta$ is defined as in (3.13).

Proof. First let $l$ be even. For $n_{0} \in \mathbb{N}$ sufficiently large let us consider the functions (note $\gamma \in \mathbb{R}^{+}$)

$$
f_{n}(z):=\frac{i \ln \left(\gamma \Delta_{n}(z)\right)}{\sqrt{z^{-l} R(z)}}, \quad z \in \mathcal{B} \backslash \Gamma_{l}, n \geq n_{0},
$$

which are well defined and analytic by Lemma 2.2 and (3.18) (note that Lemma 2.2 also holds on $\Gamma_{[0,2 \pi]} \backslash \Gamma_{l}$ by completely the same proof using Theorem 3.2 instead of Theorem 2.2). Recall that the function $\Delta$ has the representation

$$
\Delta(z)=\lim _{n \rightarrow \infty} \Delta_{n}(z)=\lim _{n \rightarrow \infty}\left(\widetilde{\Phi}_{n}^{*}(z) \mathcal{G}_{n}(z)-z \widetilde{\Phi}_{n}(z) \mathcal{H}_{n}(z)\right), \quad z \in \mathcal{B}
$$

where convergence is uniform on compact subsets of $\mathcal{B} \backslash \Gamma_{l}$ and on $\Gamma_{l}$. From (3.3a) we obtain for $\varphi \in[0,2 \pi] \backslash E_{l}$

$$
\Delta_{n}\left(e^{i \varphi}\right)=2 \operatorname{Re}\left\{\widetilde{\Phi}_{n}^{*}\left(e^{i \varphi}\right) \mathcal{G}_{n}\left(e^{i \varphi}\right)\right\}
$$

thus

$$
\ln \gamma\left(\Delta_{n}\left(e^{i \varphi}\right)\right)=\ln \left|\gamma \Delta_{n}\left(e^{i \varphi}\right)\right| \in \mathbb{R} \quad \text { for } \varphi \in[0,2 \pi] \backslash E_{l} .
$$


Since by (3.18) (compare $[15,17])$

$$
\sqrt{R\left(e^{i \varphi}\right)}= \begin{cases}(-1)^{j} i e^{i \frac{l}{2} \varphi} \sqrt{\left|R\left(e^{i \varphi}\right)\right|}, & \varphi \in\left[\varphi_{2 j-1}, \varphi_{2 j}\right], j=1, \ldots, l, \\ (-1)^{j} e^{i \frac{l}{2} \varphi} \sqrt{\left|R\left(e^{i \varphi}\right)\right|}, & \varphi \in\left[\varphi_{2 j}, \varphi_{2 j+1}\right], j=0, \ldots, l,\end{cases}
$$

we have for the radial boundary values

$$
\lim _{s \rightarrow 1^{-}} \operatorname{Re} f_{n}\left(s e^{i \varphi}\right)=\left\{\begin{array}{l}
0, \quad \varphi \in[0,2 \pi] \backslash E_{l}, \\
\frac{\ln \left|\gamma \Delta_{n}\left(e^{i \varphi}\right)\right|}{r(\varphi)}, \quad \varphi \in \operatorname{Int} E_{l} .
\end{array}\right.
$$

Note that by the uniform convergence in $((1)) \Delta$ is a continuous function on $\Gamma_{l}$ which is, by Theorem 3.3 and the assumption on $\sigma^{\prime}(\varphi) / \tilde{\sigma}^{\prime}(\varphi)$, uniformly bounded away from zero and infinity. Again by the uniform convergence in $((1))$ on $\Gamma_{l}$ the same holds true for the $\Delta_{n}$ 's and hence

$$
\frac{\ln \left|\gamma \Delta_{n}\left(e^{i \varphi}\right)\right|}{r(\varphi)} \in L_{1}\left(E_{l}\right)
$$

For a fixed $n \geq n_{0}$ and $0 \leq s<1$ let us consider the function $f_{n}(s z)$, which is analytic on $\mathcal{B}$. By the definition of the $f_{n}$ 's, by (1.29) and the first condition in (3.16) we have $f_{n}(0)=0$. Hence from [7, Ch.II.B (pp. 48-49) and Ch.I.E. (Theorem, p. 25)] we obtain

$$
f_{n}(s z)=\frac{1}{2 \pi} \int_{0}^{2 \pi} \frac{e^{i \varphi}+z}{e^{i \varphi}-z} \operatorname{Re} f_{n}\left(s e^{i \varphi}\right) d \varphi, \quad|z|<1 .
$$

Now let $s_{\nu}$ be an arbitrary sequence in $[0,1)$ with $s_{\nu} \rightarrow 1^{-}$. From $((2))-((4))$ and Lebesgue's Dominated Convergence Theorem we get

$$
f_{n}(z)=\frac{i \ln \left(\gamma \Delta_{n}(z)\right)}{\sqrt{z^{-l} R(z)}}=\frac{1}{2 \pi} \int_{E_{l}} \frac{e^{i \varphi}+z}{e^{i \varphi}-z} \frac{\ln \left|\gamma \Delta_{n}\left(e^{i \varphi}\right)\right|}{r(\varphi)} d \varphi, \quad|z|<1,
$$

and therefore

((5)) $\gamma \Delta_{n}(z)=\exp \left\{\frac{1}{4 \pi i} \sqrt{z^{-l} R(z)} \int_{E_{l}} \frac{e^{i \varphi}+z}{e^{i \varphi}-z} \frac{\ln \left|\gamma \Delta_{n}\left(e^{i \varphi}\right)\right|^{2}}{r(\varphi)} d \varphi\right\}, \quad|z|<1$.

Using again Lebesgue's Theorem we obtain from $((1)),((5))$ and (3.14)

$$
\gamma \Delta(z)=\exp \left\{\frac{1}{4 \pi i} \sqrt{z^{-l} R(z)} \int_{E_{l}} \frac{e^{i \varphi}+z}{e^{i \varphi}-z} \ln \frac{\sigma^{\prime}(\varphi)}{\tilde{\sigma}^{\prime}(\varphi)} \frac{d \varphi}{r(\varphi)}\right\} \quad \text { for }|z|<1 .
$$

Because the function at the right-hand side of (3.20a) is analytic on $\mathcal{B} \backslash \Gamma_{l}$ and $\Delta$ is analytic on $|z|<1$ and continuous on $\mathcal{B} \backslash \Gamma_{l}$, the assertion (3.20a) also holds on $\mathcal{B} \backslash \Gamma_{l}$.

If $l$ is odd we put $R_{4 l}(z):=R\left(z^{2}\right)$ and denote, as in (3.16) and (3.17), by $E_{2 l}$ the set given by $e^{-2 i l \varphi} R_{4 l}\left(e^{i \varphi}\right) \leq 0$, which consists of $2 l$ intervals. Furthermore let $a_{2 n, 2 l}:=0$ and $a_{2 n+1,2 l}:=a_{n}$ for $n \in \mathbb{N}_{0}$ and analogously, by $b_{2 n, 2 l}:=0$ and $b_{2 n+1,2 l}:=b_{n}$ for $n \in \mathbb{N}_{0}$. Then one can show that the orthogonal polynomials $\Phi_{n, 2 l}, \widetilde{\Phi}_{n, 2 l}$ associated with $\left\{a_{n, 2 l}\right\}_{n \in \mathbb{N}_{0}}$ resp. $\left\{b_{n, 2 l}\right\}_{n \in \mathbb{N}_{0}}$ can be represented in the form (see e.g. [16, Example 2.2(c)])

$$
\begin{array}{ll}
\Phi_{2 n, 2 l}(z)=\Phi_{n}\left(z^{2}\right), & \Phi_{2 n+1,2 l}(z)=z \Phi_{n}\left(z^{2}\right), \\
\widetilde{\Phi}_{2 n, 2 l}(z)=\widetilde{\Phi}_{n}\left(z^{2}\right), & \widetilde{\Phi}_{2 n+1,2 l}(z)=z \widetilde{\Phi}_{n}\left(z^{2}\right)
\end{array}
$$

and they are orthogonal with respect to

$$
\sigma_{2 l}(\varphi):=\sigma(2 \varphi), \quad \tilde{\sigma}_{2 l}(\varphi):=\tilde{\sigma}(2 \varphi) .
$$


Further the C-functions $F_{2 l}$ and $\widetilde{F}_{2 l}$ corresponding to $\sigma_{2 l}$ and $\tilde{\sigma}_{2 l}$ are related to $F$ and $\widetilde{F}$ by

$$
F_{2 l}(z)=F\left(z^{2}\right), \quad \widetilde{F}_{2 l}(z)=\widetilde{F}\left(z^{2}\right) .
$$

All the assumptions of the theorem are fulfilled for the measures $\sigma_{2 l}$ and $\tilde{\sigma}_{2 l}$; thus Theorem 3.1 leads to a function $\Delta_{2 l}$ which is, by the already proven part of this theorem, of the form

$$
\begin{aligned}
\Delta_{2 l}(z)=\gamma^{-1} \exp \left\{\frac{1}{4 \pi i} \sqrt{z^{-2 l} R_{4 l}(z)} \int_{E_{2 l}} \frac{e^{i \varphi}+z}{e^{i \varphi}-z} \ln \frac{\sigma_{2 l}^{\prime}(\varphi)}{\tilde{\sigma}_{2 l}^{\prime}(\varphi)} \frac{d \varphi}{r_{2 l}(\varphi)}\right\} \\
=\gamma^{-1} \exp \left\{\frac{1}{4 \pi i} \sqrt{\left(z^{2}\right)^{-l} R\left(z^{2}\right)} \int_{0}^{\pi}\left[\frac{e^{i \varphi}+z}{e^{i \varphi}-z}-\frac{e^{i(\varphi+\pi)}+z}{e^{i(\varphi+\pi)}-z}\right] \ln \frac{\sigma^{\prime}(2 \varphi)}{\tilde{\sigma}^{\prime}(2 \varphi)} \frac{d \varphi}{r(2 \varphi)}\right\}, \\
z \in \mathcal{B} \backslash \Gamma_{2 l},
\end{aligned}
$$

where the second equation holds by $r_{2 l}(\varphi)=r(2 \varphi)=-r(2(\varphi+\pi))$, which follows from the definition of $r_{2 l}$ in (3.19) since $l$ is odd, and by $\sigma_{2 l}(\varphi+\pi)=\sigma_{2 l}(\varphi)$, $\tilde{\sigma}_{2 l}(\varphi+\pi)=\tilde{\sigma}_{2 l}(\varphi)$ (note that $F_{2 l}$ and $\widetilde{F}_{2 l}$ are functions in $z^{2}$, i.e. the odd moments vanish). From

$$
\frac{e^{i \varphi}+z}{e^{i \varphi}-z}-\frac{e^{i(\varphi+\pi)}+z}{e^{i(\varphi+\pi)}-z}=\frac{4 z e^{i \varphi}}{e^{2 i \varphi}-z^{2}}
$$

and the above representation of $\Delta_{2 l}$ we obtain

$$
\begin{aligned}
& \Delta_{2 l}(z)=\gamma^{-1} \exp \left\{\frac{1}{2 \pi i} \sqrt{\left(z^{2}\right)^{-l} R\left(z^{2}\right)} \int_{E_{l}} \frac{z e^{i \frac{\varphi}{2}}}{e^{i \varphi}-z^{2}} \ln \frac{\sigma^{\prime}(\varphi)}{\tilde{\sigma}^{\prime}(\varphi)} \frac{d \varphi}{r(\varphi)}\right\}, \\
& z \in \mathcal{B} \backslash \Gamma_{2 l} .
\end{aligned}
$$

Now we use the fact that

$$
\Delta_{2 l}(z)=\Delta\left(z^{2}\right)
$$

which can be derived from the Theorems 2.2 and 3.1, taking into consideration the relations $((6))$ and $((7))$ and the relation

$\mathcal{G}_{2 n, 2 l}(z)=\mathcal{G}_{2 n+1,2 l}(z)=\mathcal{G}_{n}\left(z^{2}\right), \quad \mathcal{H}_{2 n, 2 l}(z)=z \mathcal{H}_{n}\left(z^{2}\right), \quad \mathcal{H}_{2 n+1,2 l}(z)=\mathcal{H}_{n}\left(z^{2}\right)$, i.e. $\Delta_{2 n, 2 l}(z)=\Delta_{2 n+1,2 l}(z)=\Delta_{n}\left(z^{2}\right)$. Substituting $z^{2}=w$ in $((8))$, representation $(3.20 \mathrm{~b})$ is proved.

From Theorem 2.2, Theorem 3.2 and Theorem 3.4 we immediately get

Corollary 3.1. Under the assumptions of Theorem 3.4 there holds

$\lim _{n \rightarrow \infty} \frac{\widetilde{\Phi}_{n}^{*}(z)}{\Phi_{n}^{*}(z)}=\left\{\begin{array}{l}\beta^{-1} \exp \left\{\frac{1}{4 \pi i} \sqrt{z^{-l} R(z)} \int_{E_{l}} \frac{e^{i \varphi}+z}{e^{i \varphi}-z} \ln \frac{\sigma^{\prime}(\varphi)}{\tilde{\sigma}^{\prime}(\varphi)} \frac{d \varphi}{r(\varphi)}\right\} \\ \beta^{-1} \exp \left\{\frac{1}{2 \pi i} \sqrt{z^{-l+1} R(z)} \int_{E_{l}} \frac{e^{i \frac{\varphi}{2}}}{e^{i \varphi}-z} \ln \frac{\sigma^{\prime}(\varphi)}{\tilde{\sigma}^{\prime}(\varphi)} \frac{d \varphi}{r(\varphi)}\right\} \quad \text { if } l \text { is is odd }\end{array}\right.$ uniformly on each compact subset of $\mathcal{B} \backslash \Gamma_{l}$.

\section{ACKNOWLEDGMENT}

We would like to thank the referee for a careful reading of the manuscript and for his valuable comments. 


\section{REFERENCES}

1. N. I. Akhiezer, On polynomials on a circular arc, Soviet Math. Dokl. 1 (1960), 31-34.

2. G. Freud, Orthogonal polynomials, Pergamon Press, 1971.

3. Ya. L. Geronimus, On the character of the solutions of the moment problem in the case of a limitperiodic associated fraction, Izv. Akad. Nauk SSSR Ser. Mat. 5 (1941), 203-210. MR 3:110b

4. , Polynomials orthogonal on a circle and interval, Pergamon Press, New York, 1960. MR 24:A3468

5. _ Orthogonal polynomials, Consultants Bureau,New York, 1961. MR 40:2872

6. Polynomials orthogonal on a circle and their applications, Amer. Math. Soc. Transl. 3 (1962), 1-78.

7. P. Koosis, Introduction to $H_{p}$ spaces, London Mathematical Society, Lecture Note Series, Vol. 40, Cambridge Univ. Press, 1980. MR 81c:30062

8. A. Maté, P. Nevai, and V. Totik, Extensions of Szegö's theory of orthogonal polynomials, II, Constr. Approx. 3 (1987), 51-72. MR 88m:42044a

9. Extensions of Szegö's theory of orthogonal polynomials, III, Constr. Approx. 3 (1987), 73-96. MR 88m:42044a

10. _ Szegö's extremum problem on the unit circle, Ann. of Math. 134 (1991), 433-453. MR 92i: 42014

11. P. Nevai and W. Van Assche, Compact perturbations of orthogonal polynomials, Pacific J. Math. 153 (1992), 163-184. MR 93c:42022

12. L. Golinskii, P. Nevai, and W. Van Assche, Perturbation of orthogonal polynomials on an arc of the unit circle J. Approx. Theory (to appear).

13. F. Peherstorfer, On the asymptotic behaviour of functions of the second kind and Stieltjes polynomials and on Gauss-Kronrod quadrature formulas, J. Approx. Theory 70 (1992), 156190. MR 93h: 42020

14. _ A special class of polynomials orthogonal on the unit circle including the associated polynomials, Constr. Approx. (to appear).

15. F. Peherstorfer and R. Steinbauer, Orthogonal polynomials on arcs of the unit circle, I, J. Approx. Theory (to appear).

16. Characterization of orthogonal polynomials with respect to a functional, J. Comput. Appl. Math. (to appear).

17. _ Asymptotic behaviour of orthogonal polynomials with asymptotically periodic recurrence coefficients, J. Approx. Theory (to appear).

18. R. Steinbauer, Orthogonalpolynome auf mehreren Bögen des komplexen Einheitskreises, Ph.D. dissertation, Universitätsverlag Rudolf Trauner, Linz, 1995.

19. E. A. Rakhmanov, On the asymptotics of the ratio of orthogonal polynomials, Math. USSR-Sb. 32 (1977), 199-213.

20. (1983), 105-117.

21. On asymptotics of polynomials orthogonal on the unit circle with weights not satisfying Szegö's condition, Math. USSR-Sb. 58 (1987), 149-167.

22. G. Szegö, Orthogonal polynomials, 4th ed., Amer. Math. Soc. Colloq. Publ., Vol. 23, Amer. Math. Soc., Providence, R.I., 1975. MR 51:8724

23. W. Van Assche, Asymptotics for orthogonal polynomials and three-term recurrences, Orthogonal polynomials: Theory and Practice (P. Nevai, ed.), NATO ASI Series C, vol. 294, Kluwer, Dordrecht, 1990, pp. 435-462. MR 92k:42038

Institut für Mathematik, Johannes Kepler Universität Linz, A-4040 Linz, Austria

E-mail address: franz.peherstorfer@jk.uni-linz.ac.at

E-mail address: robert.steinbauer@jk.uni-linz.ac.at 